$\mathbf{s}$

tha 5 (12)

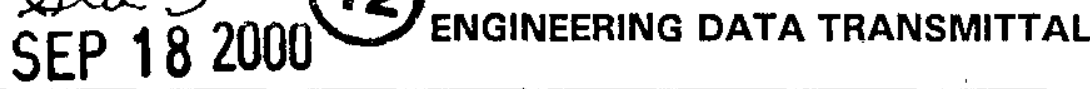

Page 1 of _ _

1. EDT 628337
2. To: (Receiving Organization)

Distribution

5. Proj./Prog./Dept./Div.

PEP Analytical Laboratory

8. Originator Remarks:

Initial Release and Distribution

Key Words: SigmaPlot, moisture, water, analysis, supercritical

fluid, FTIR

11. Receiver Remarks:

11A. Design Baseline Document? $O$ Yes $O$ No

\section{From: (Originating Organization) \\ Plutonium Finishing Plant}

6. Design Authority/Design Agent/Cog. Engr.:

ST Hurlbut

\section{Related EDT No.: \\ $\mathrm{N} / \mathrm{A}$ \\ 7. Purchase Order No.: \\ $\mathrm{N} / \mathrm{A}$ \\ 9. Equip./Component No.: \\ $\mathrm{N} / \mathrm{A}$}

10. System/BIdg./Facility:

PFP

12. Major Assm. Dwg. No.:

$\mathrm{N} / \mathrm{A}$

13. Permit/Permit Application No.:

$\mathrm{N} / \mathrm{A}$

14. Required Response Date:

$\mathrm{N} / \mathrm{A}$

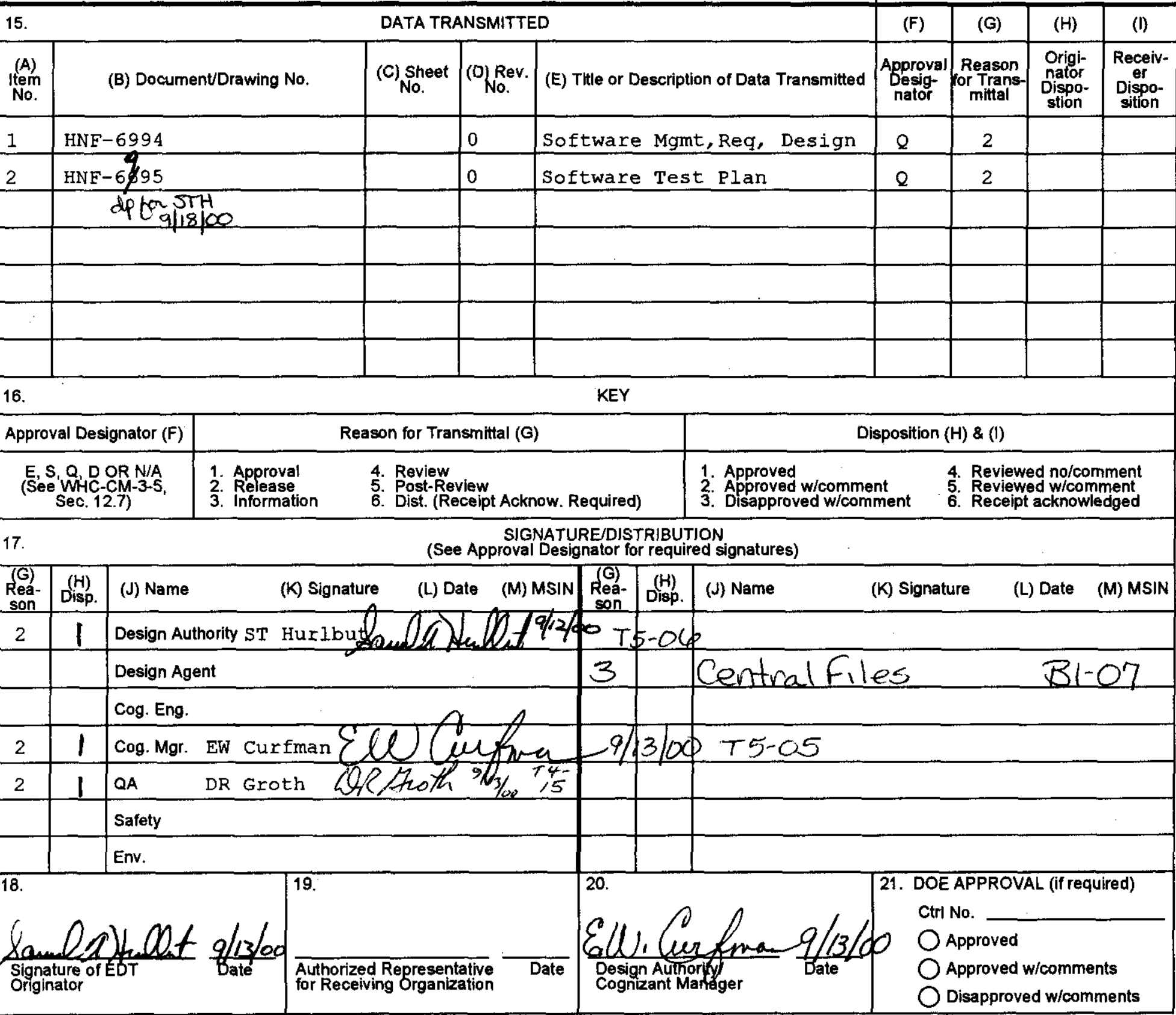


HNF-6995

Revision 0

\section{SigmaPlot 2000, Version 6.00, SPSS Inc. Computer Software Test Plan}

Prepared for the U.S. Department of Energy

Assistant Secretary for Environmental Management

Project Hanford Management Contractor for the

U.S. Department of Energy under Contract DE-AC06-96RL13200

Fluor Hanford

P.O. Box 1000

Richland, Washington 
HNF-6995

Revision 0

EDT-628337

\title{
SigmaPlot 2000, Version 6.00, SPSS Inc. \\ Computer Software Test Plan
}

\author{
S. T. Hurlbut \\ Fluor Hanford
}

Date Published

September 2000

Prepared for the U.S. Department of Energy

Assistant Secretary for Environmental Management

Project Hanford Management Contractor for the

U.S. Department of Energy under Contract DE-AC06-96RL13200

Fluor Hanford

P.O. Box 1000

Richland, Washington

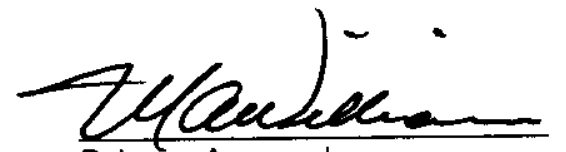

Release Approval

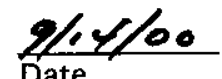

Date

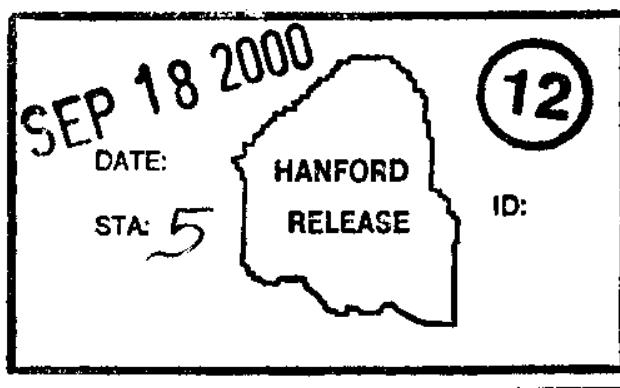

Release Stamp 
HNF-6995

Revision 0

TRADEMARK DISCLAIMER

Reference herein to any specific commercial product, process,

or service by trade name, trademark, manufacturer, or otherwise, does not necessarily constitute or imply its

endorsement, recommendation, or favoring by the United

States Government or any agency thereof or its contractors or subcontractors.

This report has been reproduced from the best available copy.

Printed in the United States of Annerica

Total Pages: 39

* SigmaPlot is a registered trademark of Jandel Corporation. 


\section{PLUTONIUM FINISHING PLANT ANALYTICAL LABORATORY}

\section{SigmaPlot 2000, Version 6.00, SPSS, Inc. Computer Software Test Plan}
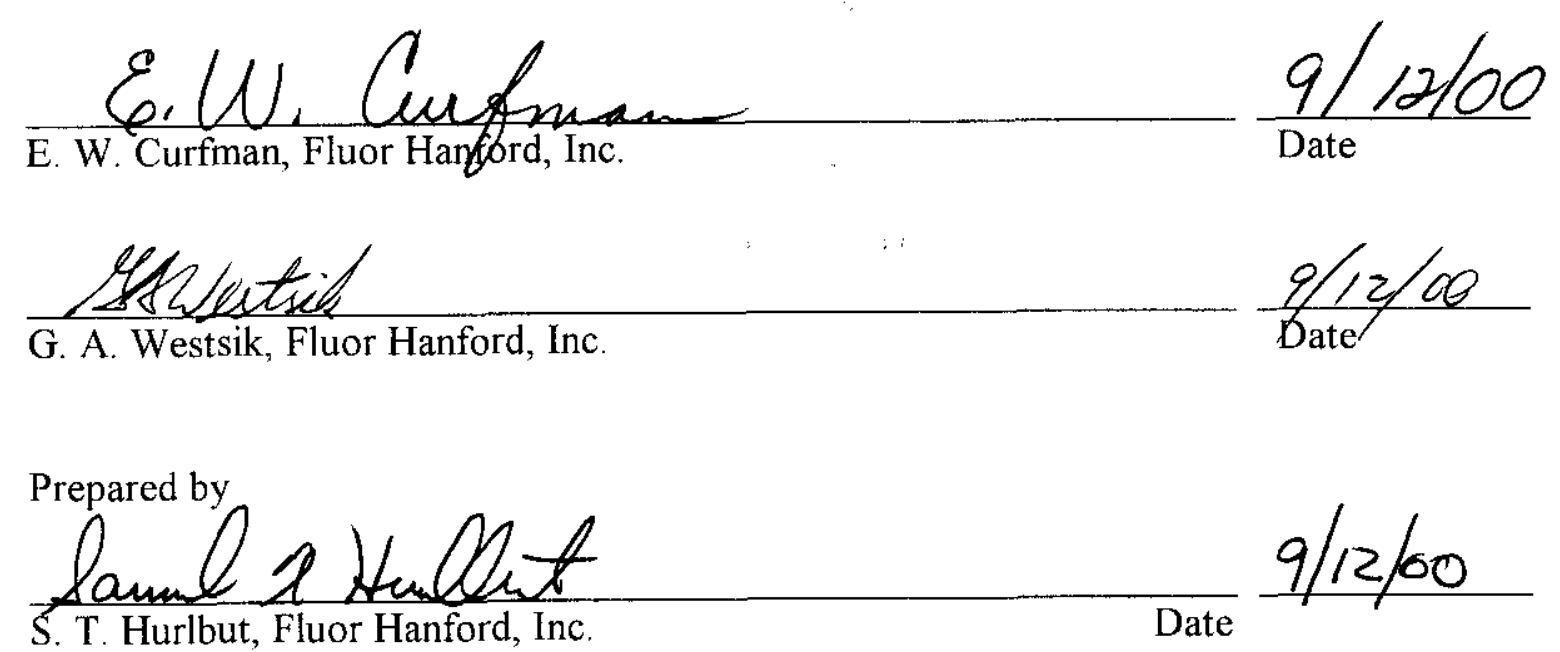

Date $9 / 12 / 00$ 


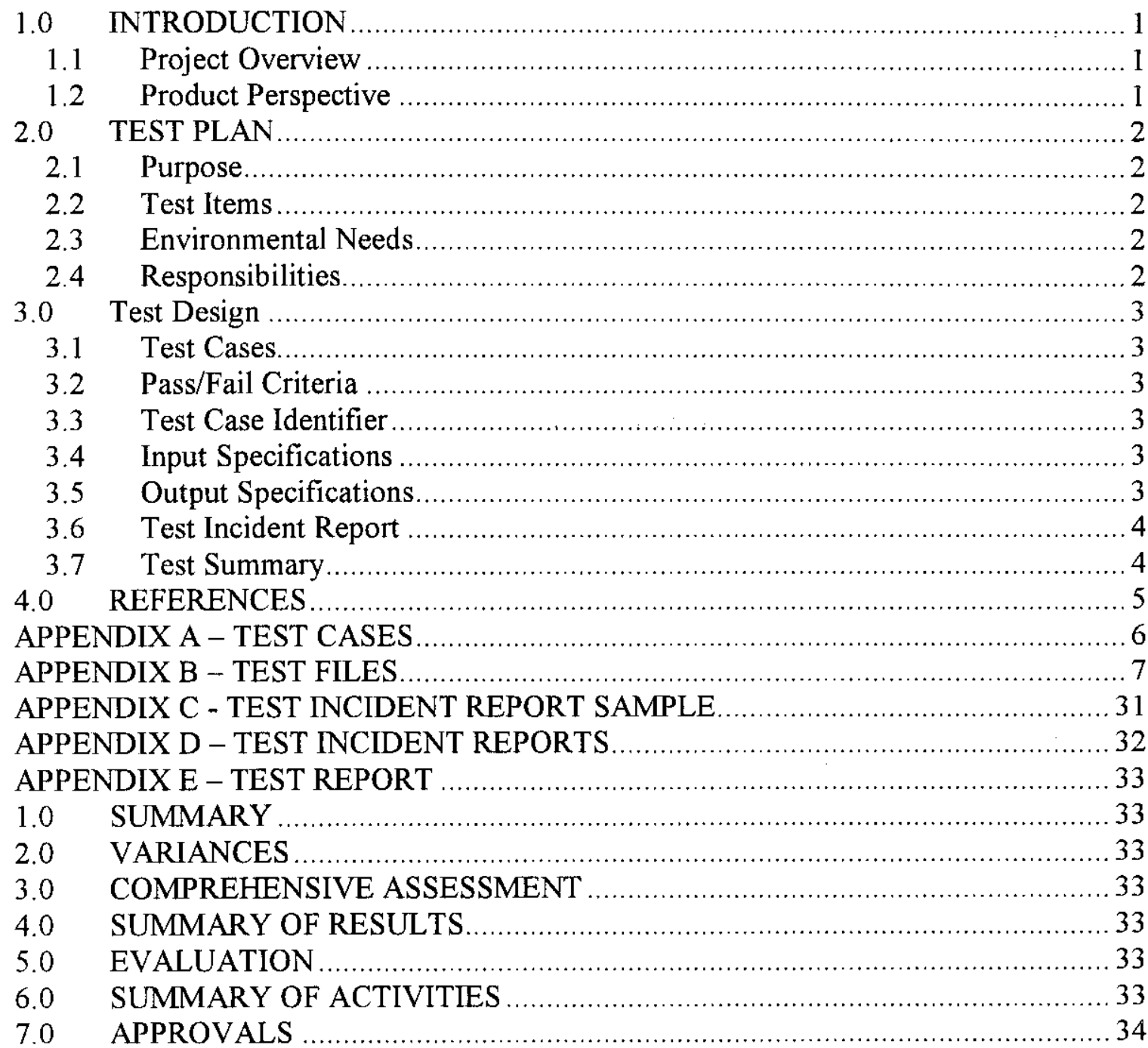




\section{$1.0 \quad$ INTRODUCTION}

\subsection{Project Overview}

SigmaPlot is a vendor software product used in conjunction with the supercritical fluid extraction Fourier transform infrared spectrometer (SFE-FTIR) system. This product converts the raw spectral data to useful area numbers. SigmaPlot will be used in conjunction with procedure ZA-565-301, "Determination of Moisture by Supercritical Fluid Extraction and Infrared Detection."

\subsection{Product Perspective}

SigmaPlot is a proprietary program provided by the SPSS, Inc. 


\section{$2.0 \quad$ TEST PLAN}

\subsection{Purpose}

This test plan will be performed in conjunction with or prior to HNF-6936, "HA-53 Supercritical Fluid Extraction System Acceptance Test Plan", to perform analyses for water. The test will ensure that the software can be installed properly and will manipulate the analytical data correctly.

\section{$2.2 \quad$ Test Items}

This test document is specifically written to test the installation and operation of the SigmaPlot software in connection to the MIDAC FTIR.

\subsection{Environmental Needs}

SigmaPlot will be installed on a computer meeting the hardware requirements in the MIDAC User's Guide including the MIDAC supplied interface board. The computer will be connected to the MIDAC FTIR.

\section{$2.4 \quad$ Responsibilities}

The scientist responsible for the analytical procedure, ZA-565-301, shall be responsible for preparing the test and resolving any test-related issues. The test shall be witnessed by a PFPAL team leader/manager. 


\subsection{Test Design}

\subsection{Test Cases}

Test cases and their test items will be executed in the order they are numbered. Results of the testing process, including visually observable results (error message generated, aborts, and requests for operation action) and notes on the successful execution of the test will be documented in each test case; Pass/Fail notes will be indicated on the test cases. Test cases are included in Appendix A of this document.

Test incidents, with the disposition of any anomalies discovered during testing, will be documented. Test problems/deficiencies/defects need to be noted on a test incident report, indicating the specific item where the problem occurred. Test incidents are integral to the test cases and will be kept with the record copies of the test cases. A sample test incident report is included in Appendix B. Actual test incident reports will be included in Appendix C.

At the successful completion of all test cases, a Test Summary report will be prepared. The Test Summary Report is included in Appendix D.

\subsection{Pass/Fail Criteria}

Acceptance criteria state the conditions that must be satisfied by the system or product before the user formally accepts the system. If a test case achieves all of its requirements, it is considered a PASS. If a test case does not achieve all of its requirements, it is considered a FAIL and must be corrected and retested. If the test produces a result that is not desirable by the user or the user's management and it meets the requirements detailed in the test case, a decision must be made to either change the requirement and retest, or PASS the test.

\subsection{Test Case Identifier}

All test cases will be uniquely identified by a test case number. Each test item will also be numbered to provide a means of identifying anomalies.

\subsection{Input Specifications}

Test data will be developed or provided to thoroughly test all areas of the system. Where possible, this data will be identified in such a way that the correct outcome is known in advance. Effectiveness of the data will be ensured through joint participation by user personnel. Transactions that are specifically designed to check internal controls and decision logic will be included.

\subsection{Output Specifications}


All elements within the system will meet or exceed the following requirements, as stated in the test cases.

- File data will be of documented length and type.

- Screens will be formatted and display/update data as documented.

- Reports will present data consistent with stated requirements.

\subsection{Test Incident Report}

Test incidents will be reported using the Test Incident report (See Appendix B). Required information is as follows:

- Test Case and test item numbers

- Date encountered

- Expected results

- Actual results

- Tester name

- Witness (if required)

These forms will be followed up with the users, users' manager and responsible scientist to determine course of action, which may include correction to the software or change to the requirement.

\subsection{Test Summary}

At the completion of the testing process, a test summary report will be completed (Appendix C). This report will evaluate and summarize the test results including the following:

- Identify the system and the items tested, indicating their revision identification.

- Indicate the environment in which the testing activities took place.

- State whether or not the system is ready for operation.

- Supply references to the following documentation if they exist: test plan, test design, test cases, and test incident report.

This Summary will also include a Test Verification Sheet to be signed by the appropriate persons to indicate the final validation and acceptance of the system. 


\subsection{REFERENCES}

SigmaPlot User's Guide, SPSS, Inc.

ZA-565-301, Determination of Moisture by Supercritical Fluid Extraction and Infrared Detection, Fluor Hanford, Inc., Richland, WA

HNF-6936, HA-53 Supercritical Fluid Extraction System Acceptance Test Plan, Fluor Hanford, Inc., Richland, WA 


\section{APPENDIX A - TEST CASES}

\begin{tabular}{|c|c|c|c|c|}
\hline $\begin{array}{l}\text { TEST } \\
\text { STEP }\end{array}$ & ACTION & EXPECTED RESULT & $\begin{array}{l}\text { PASS/ } \\
\text { FAIL }\end{array}$ & $\begin{array}{l}\text { P/F ON } \\
\text { RETEST }\end{array}$ \\
\hline 1. & $\begin{array}{l}\text { Install software per vendor } \\
\text { instructions. }\end{array}$ & The software installs. & & \\
\hline 2. & $\begin{array}{l}\text { Import text file } \\
\text { (HNF6995.txt) containing } \\
\text { spectral data. } \\
\end{array}$ & $\begin{array}{l}\text { File is imported without } \\
\text { incident. }\end{array}$ & & \\
\hline 3. & $\begin{array}{l}\text { Edit file to eliminate data } \\
\text { taken prior to } \mathrm{CO}_{2} \text { flow. }\end{array}$ & Rows are deleted. & & \\
\hline 4. & Perform area calculation & $\begin{array}{l}\text { Relative area will be } \\
\text { calculated. }\end{array}$ & & \\
\hline 5. & $\begin{array}{l}\text { Import text file } \\
\text { (HNF6995x2.txt) } \\
\text { containing modified }(x 2) \\
\text { spectral data. } \\
\end{array}$ & $\begin{array}{l}\text { File is imported without } \\
\text { incident. }\end{array}$ & & \\
\hline 6. & $\begin{array}{l}\text { Edit file to eliminate data } \\
\text { taken prior to } \mathrm{CO}_{2} \text { flow. }\end{array}$ & $\begin{array}{l}\text { Rows and columns are } \\
\text { deleted. }\end{array}$ & & \\
\hline 7. & Perform area calculation & $\begin{array}{l}\text { Calculated area will be two } \\
\text { times result from test } 4 \text {. }\end{array}$ & & \\
\hline 8. & $\begin{array}{l}\text { Import text file } \\
\text { (HNF6995x5.txt) } \\
\text { containing modified }(x 5) \\
\text { spectral data. } \\
\end{array}$ & $\begin{array}{l}\text { File is imported without } \\
\text { incident. }\end{array}$ & & \\
\hline 9. & $\begin{array}{l}\text { Edit file to eliminate data } \\
\text { taken prior to } \mathrm{CO}_{2} \text { flow. }\end{array}$ & $\begin{array}{l}\text { Rows and columns are } \\
\text { deleted. }\end{array}$ & & \\
\hline 10. & Perform area calculation & $\begin{array}{l}\text { Calculated area will be five } \\
\text { times result from test } 4 \text {. }\end{array}$ & & \\
\hline
\end{tabular}

Tester Initials (this should appear on each test case page) 


\section{APPENDIX B - TEST FILES}

\section{B-1 \\ Data to be used in test case 2 .}

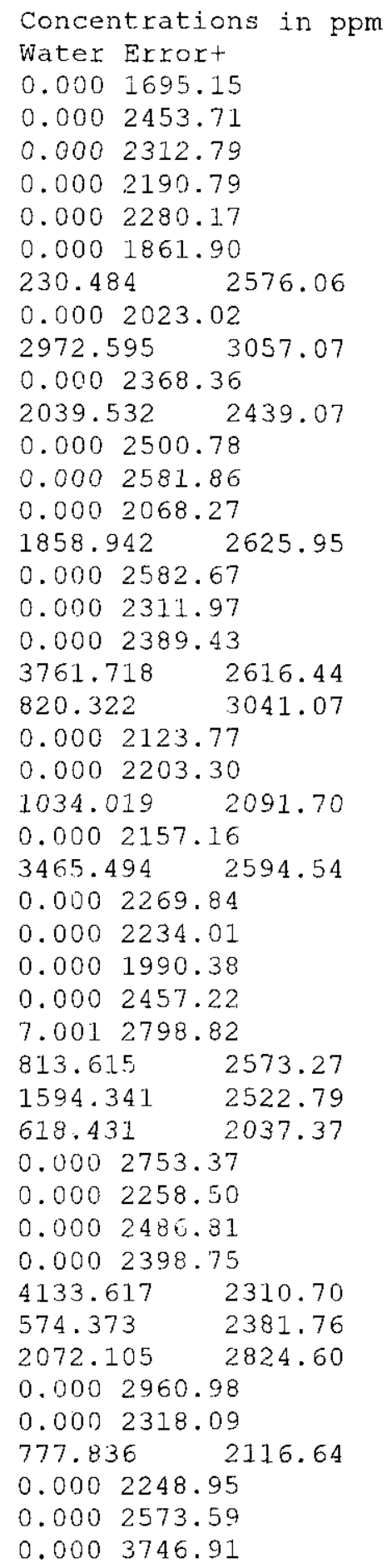




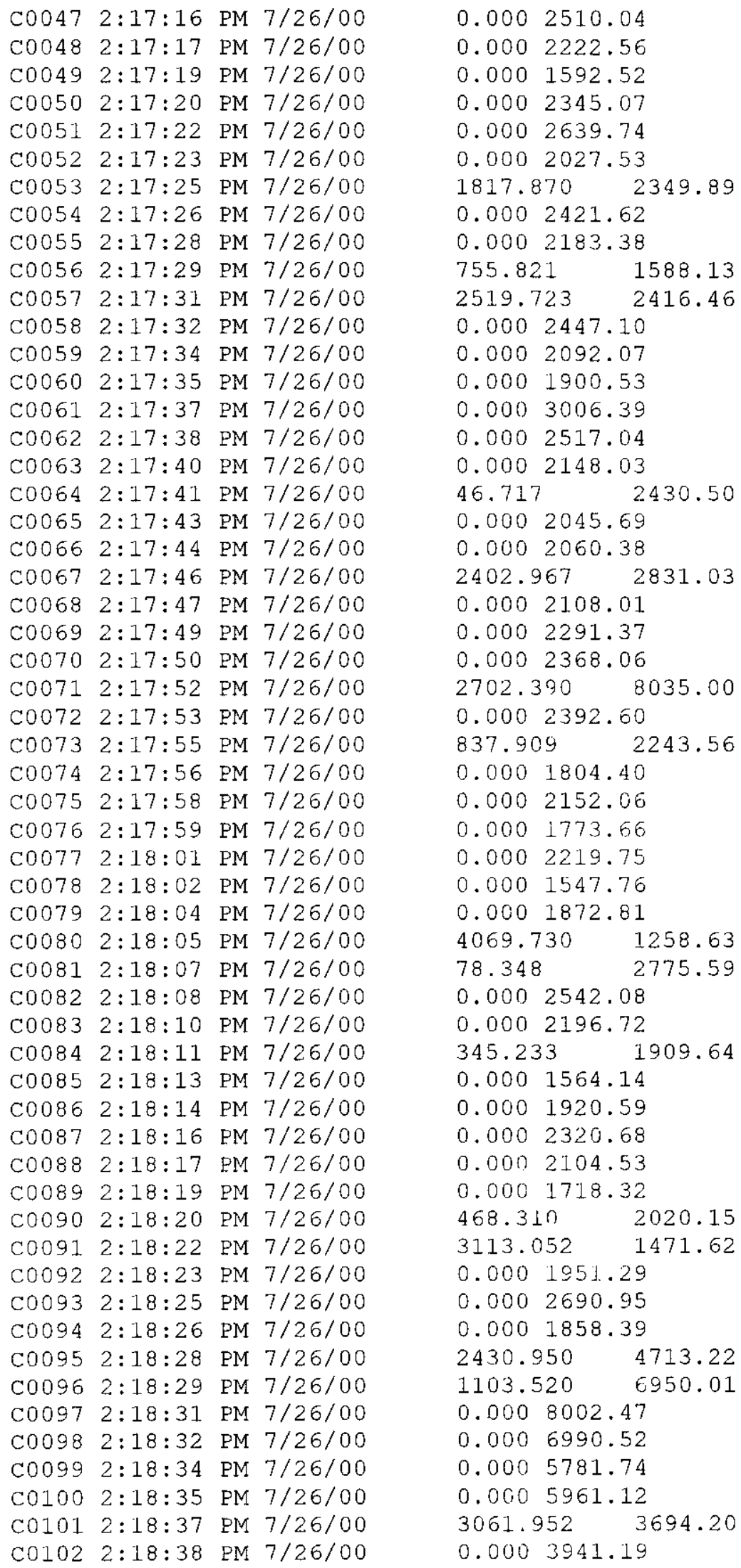

C0048 2:17:17 PM 7/26/00

C0049 2:17:19 PM 7/26/00

C0050 2:17:20 PM 7/26/00

C0051 2:17:22 PM 7/26/00

C0052 2:17:23 PM 7/26/00

C0053 2:17:25 PM 7/26/00

C0054 2:17:26 PM 7/26/00

C0055 2:17:28 PM 7/26/00

C0056 2:17:29 PM 7/26/00

C0057 2:17:31 PM 7/26/00

C0058 2:17:32 PM 7/26/00

C0059 2:17:34 PM 7/26/00

C0060 2:17:35 PM 7/26/00

C0061 2:17:37 PM 7/26/00

C0062 2:17:38 PM 7/26/00

C0063 2:17:40 PM 7/26/00

C0064 2:17:41 PM 7/26/00

C0065 2:17:43 PM 7/26/00

C0066 2:17:44 PM 7/26/00

C0067 2:17:46 PM 7/26/00

C0068 2:17:47 PM 7/26/00

C0069 2:17:49 PM 7/26/00

C0070 2:17:50 PM 7/26/00

C0071 2:17:52 PM 7/26/00

C0072 2:17:53 PM 7/26/00

C0073 2:17:55 PM 7/26/00

C0074 2:17:56 PM 7/26/00

C0075 2:17:58 PM 7/26/00

C0076 2:17:59 PM 7/26/00

C0077 2:18:01 PM 7/26/00

C0078 2:18:02 PM 7/26/00

C0079 2:18:04 PM 7/26/00

C0080 2:18:05 PM 7/26/00

C0081 2:18:07 PM 7/26/00

C0082 2:18:08 PM 7/26/00

C0083 2:18:10 PM 7/26/00

C0084 2:18:11 PM 7/26/00

C0085 2:18:13 PM 7/26/00

C0086 2:18:14 PM 7/26/00

C0087 2:18:16 PM 7/26/00

C0088 2:18:17 PM 7/26/00

C0089 2:18:19 PM 7/26/00

C0090 2:18:20 PM 7/26/00

C0091 2:18:22 PM 7/26/00

C0092 2:18:23 PM 7/26/00

C0093 2:18:25 PM 7/26/00

C0094 2:18:26 PM 7/26/00

C0095 2:18:28 PM 7/26/00

C0096 2:18:29 PM 7/26/00

C0097 2:18:31 PM 7/26/00

C0098 2:18:32 PM 7/26/00

C0099 2:18:34 PM 7/26/00

$\mathrm{C} 0100$ 2:18:35 PM 7/26/00

C0101 2:18:37 PM 7/26/00

C0102 2:18:38 PM 7/26/00

0.0002222 .56

$0.000 \quad 1592.52$

0.0002345 .07

$0.000 \quad 2639.74$

$0.000 \quad 2027.53$

$1817.870 \quad 2349.89$

0.0002421 .62

0.0002183 .38

$755.821 \quad 1588.13$

$2519.723 \quad 2416.46$

0.0002447 .10

0.0002092 .07

0.0001900 .53

0.0003006 .39

0.0002517 .04

0.0002148 .03

$46.717 \quad 2430.50$

0.0002045 .69

0.0002060 .38

$2402.967 \quad 2831.03$

0.0002108 .01

$0.000 \quad 2291.37$

0.0002368 .06

$2702.390 \quad 8035.00$

$0.000 \quad 2392.60$

$837.909 \quad 2243.56$

0.0001804 .40

0.0002152 .06

$0.000 \quad 1773.66$

0.0002219 .75

0.0001547 .76

0.0001872 .81

$4069.730 \quad 1258.63$

$78.348 \quad 2775.59$

0.0002542 .08

0.0002196 .72

$345.233 \quad 1909.64$

$0.000 \quad 1564.14$

$0.000 \quad 1920.59$

$0.000 \quad 2320.68$

0.0002104 .53

$0.000 \quad 1718.32$

$468.310 \quad 2020.15$

$3113.052 \quad 1471.62$

0.0001951 .29

0.0002690 .95

0.0001858 .39

$2430.950 \quad 4713.22$

$1103.520 \quad 6950.01$

0.0008002 .47

$0.000 \quad 6990.52$

0.0005781 .74

$0.000 \quad 5961.12$

$3061.952 \quad 3694.20$

0.0003941 .19 
C0103 2:18:40 PM 7/26/00

C0104 2:18:41 PM 7/26/00

C0105 2:18:43 PM 7/26/00

C0106 2:18:44 PM 7/26/00

C0107 2:18:46 PM 7/26/00

C0108 2:18:47 PM 7/26/00

C0109 2:18:50 PM 7/26/00

C0110 2:18:52 PM 7/26/00

C0111 2:18:53 PM 7/26/00

C0112 2:18:55 PM 7/26/00

C0113 2:18:56 PM 7/26/00

C0114 2:18:58 PM 7/26/00

C0115 2:18:59 PM 7/26/00

C0116 2:19:01 PM 7/26/00

C0117 2:19:02 PM 7/26/00

C0118 2:19:04 PM 7/26/00

C0119 2:19:05 PM 7/26/00

C0120 2:19:07 PM 7/26/00

C0121 2:19:08 PM 7/26/00

C0122 2:19:10 PM 7/26/00

C0123 2:19:11 PM 7/26/00

C0124 2:19:13 PM 7/26/00

C0125 2:19:14 PM 7/26/00

C0126 2:19:16 PM 7/26/00

C0127 2:19:17 PM 7/26/00

C0128 2:19:19 PM 7/26/00

C0129 2:19:20 PM 7/26/00

C0130 2:19:22 PM 7/26/00

C0131 2:19:23 PM 7/26/00

C0132 2:19:25 PM 7/26/00

C0133 2:19:26 PM 7/26/00

C0134 2:19:28 PM 7/26/00

C0135 2:19:29 PM 7/26/00

C0136 2:19:31 PM 7/26/00

C0137 2:19:32 PM 7/26/00

C0138 2:19:33 PM 7/26/00

C0139 2:19:35 PM 7/26/00

C0140 2:19:36 PM 7/26/00

$\mathrm{CO141} 2: 19: 38 \mathrm{PM} 7 / 26 / 00$

C0142 2:19:39 PM 7/26/00

C0143 2:19:41 PM 7/26/00

$\mathrm{C} 01442: 19: 42 \mathrm{PM} \mathrm{7/26/00}$

C0145 2:19:44 PM 7/26/00

C0146 2:19:45 PM 7/26/00

C0147 2:19:47 PM 7/26/00

C0148 2:19:48 PM 7/26/00

C0149 2:19:50 PM 7/26/00

C0150 2:19:51 PM 7/26/00

C0151 2:19:53 PM 7/26/00

C0152 2:19:54 PM 7/26/00

C0153 2:19:56 PM 7/26/00

C0154 2:19:57 PM 7/26/00

C0155 2:19:59 PM 7/26/00

C0156 2:20:00 PM 7/26/00

C0157 2:20:02 PM 7/26/00

C0158 2:20:03 EM 7/26/00
$2497.704 \quad 2826.68$

$0.000 \quad 1519.57$

$46.413 \quad 2840.60$

0.0002192 .68

$311.112 \quad 1868.83$

$24384.007 \quad 2647.81$

$18781.350 \quad 2936.09$

$19121.667 \quad 3961.02$

$21398.484 \quad 3448.65$

$19411.172 \quad 2899.09$

$18841.415 \quad 2793.44$

$16829.492 \quad 2906.88$

$17878.558 \quad 3657.23$

$16750.013 \quad 2896.06$

$16427.964 \quad 2924.80$

$15228.719 \quad 3452.61$

$18299.588 \quad 2902.45$

$17870.015 \quad 2532.09$

$20551.112 \quad 2927.84$

$23395.882 \quad 3979.40$

$29106.700 \quad 3505.07$

$27978.167 \quad 3145.46$

$26313.702 \quad 2985.40$

30782.452 .4037 .43

$34144.091 \quad 3839.62$

$27999.421 \quad 3754.30$

$27881.291 \quad 2844.22$

$29457.015 \quad 3629.77$

$24997.803 \quad 3382.96$

$28474.980 \quad .3787 .61$

$27946.425 \quad 2992.75$

$28949.475 \quad 3699.72$

$33179.686 \quad 3648.46$

$28382.191 \quad 4176.71$

$30818.047 \quad 4110.75$

$31647.103 \quad 3999.12$

$28905.829 \quad 3870.79$

$25456.795 \quad 3447.89$

$29340.508 \quad 4141.14$

$29025.481 \quad 4141.64$

$24877.349 \quad 2802.05$

$28624.272 \quad 3714.42$

$23688.702 \quad 3965.29$

$25487.462 \quad 3545.44$

$26065.655 \quad 4074.37$

$22528.230 \quad 3313.40$

$21632.604 \quad 3555.70$

$27602.564 \quad 3421.60$

$26924.306 \quad 3265.41$

$28163.663 \quad 4689.83$

$20230.430 \quad 3372.25$

$26396.573 \quad 2805.49$

$26505.297 \quad 3332.41$

$24730.738 \quad 3850.73$

$30310.748 \quad 3721.60$ 
C0159 2:20:05 PM 7/26/00

C0160 2:20:06 PM 7/26/00

C0161 2:20:08 PM 7/26/00

C0162 2:20:09 PM 7/26/00

C0163 2:20:11 PM 7/26/00

C0164 2:20:12 PM 7/26/00

C0165 2:20:14 PM 7/26/00

C0166 2:20:15 PM 7/26/00

C0167 2:20:17 PM 7/26/00

C0168 2:20:18 PM 7/26/00

C0169 2:20:20 PM 7/26/00

C0170 2:20:21 PM 7/26/00

C0171 2:20:23 PM 7/26/00

C0172 2:20:24 PM 7/26/00

C0173 2:20:26 PM 7/26/00

C0174 2:20:27 PM 7/26/00

C0175 2:20:29 PM 7/26/00

C0176 2:20:30 PM 7/26/00

C0177 2:20:32 PM 7/26/00

C0178 2:20:33 PM 7/26/00

C0179 2:20:35 PM 7/26/00

C0180 2:20:36 PM 7/26/00

C0181 2:20:38 PM 7/26/00

C0182 2:20:39 PM 7/26/00

C0183 2:20:41 PM 7/26/00

C0184 2:20:42 PM 7/26/00

C0185 2:20:44 PM 7/26/00

C0186 2:20:45 PM 7/26/00

C0187 2:20:47 PM 7/26/00

C0188 2:20:48 PM 7/26/00

C0189 2:20:50 PM 7/26/00

C0190 2:20:51 PM 7/26/00

C0191 2:20:53 PM 7/26/00

C0192 2:20:54 PM 7/26/00

C0193 2:20:56 PM 7/26/00

C0194 2:20:57 PM 7/26/00

C0195 2:20:59 PM 7/26/00

C0196 2:21:00 PM 7/26/00

C0197 2:21:02 PM 7/26/00

C0198 2:21:03 PM 7/26/00

C0199 2:21:05 PM 7/26/00

C0200 2:21:06 PM 7/26/00

C0201 2:21:08 PM 7/26/00

C0202 2:21:09 PM 7/26/00

C0203 2:21:11 PM 7/26/00

C0204 2:21:12 PM 7/26/00

C0205 2:21:14 PM 7/26/00

C0206 2:21:15 PM 7/26/00

C0207 2:21:17 PM 7/26/00

C0208 2:21:18 PM 7/26/00

C0209 2:21:20 PM 7/26/00

C0210 2:21:21 PM 7/26/00

C0211 2:21:23 PM 7/26/00

C0212 2:21:24 PM 7/26/00

C0213 2:21:26 PM 7/26/00

C0214 2:21:27 PM 7/26/00

$\begin{array}{ll}27512.395 & 3546.45 \\ 21292.237 & 3142.44 \\ 30549.359 & 3448.92 \\ 28726.496 & 3375.04 \\ 26662.919 & 3670.30 \\ 32222.098 & 4153.46 \\ 28527.035 & 3487.87 \\ 27603.301 & 4222.30 \\ 26791.411 & 3678.28 \\ 22921.546 & 4000.47 \\ 26793.242 & 3615.21 \\ 26799.504 & 4153.96 \\ 28179.089 & 3948.62 \\ 25880.803 & 3793.50 \\ 26534.100 & 3552.78 \\ 24354.770 & 3221.18 \\ 26705.775 & 3409.30 \\ 28199.597 & 2983.12 \\ 31976.815 & 4144.30 \\ 26535.811 & 3596.24 \\ 24202.140 & 3254.32 \\ 25038.962 & 2945.74 \\ 27013.999 & 2953.62 \\ 25741.572 & 3077.23 \\ 22237.767 & 3887.23 \\ 26551.024 & 3727.47 \\ 23233.344 & 3289.86 \\ 28364.800 & 3257.36 \\ 26364.636 & 3839.27 \\ 27462.781 & 3380.21 \\ 28633.429 & 3413.47 \\ 26589.240 & 3017.29 \\ 24081.437 & 3500.86 \\ 27575.961 & 3906.04 \\ 25445.649 & 3028.10 \\ 23899.957 & 2963.55 \\ 25525.367 & 3253.78 \\ 30321.843 & 3952.16 \\ 25882.304 & 3470.53 \\ 27283.950 & 3931.53 \\ 30234.259 & 3245.29 \\ 27932.652 & 3658.87 \\ 28321.984 & 3356.50 \\ 31766.798 & 4245.77 \\ 29649.106 & 3598.41 \\ 31006.998 & 3859.19 \\ 31059.607 & 3606.27 \\ 26394.913 & 4077.87 \\ 30574.801 & 3545.75 \\ 28032.450 & 3674.16 \\ 25893.487 & 3390.70 \\ 32742.003 & 4145.90 \\ 33738.016 & 3924.32 \\ 30354.086 & 4137.44 \\ 32650.069 & 4329.52 \\ 30437.743 & 3957.82\end{array}$




\begin{tabular}{|c|c|c|c|c|c|}
\hline 1 & $2: 22: 53$ & $P M$ & & 323 & 31 \\
\hline 272 & $2: 22: 54$ & PM & $7 / 26 / 00$ & 31968.142 & 3924.58 \\
\hline 273 & $2: 22: 56$ & PM & $7 / 26 / 00$ & 28428.147 & 3869.81 \\
\hline 274 & $2: 22: 57$ & PM & $7 / 26 / 00$ & 32198.447 & 4250.69 \\
\hline 0275 & $2: 22: 59$ & PM & $7 / 26 / 00$ & 24937.314 & 3731.80 \\
\hline 0276 & $2: 23: 00$ & PM & $7 / 26 / 00$ & 29024.430 & 3458.79 \\
\hline 277 & $2: 23: 02$ & PM & $7 / 26 / 00$ & 25582.001 & 3874.96 \\
\hline 278 & $2: 23: 03$ & PM & $7 / 26 / 00$ & 27403.834 & 3507.06 \\
\hline 279 & $2: 23: 05$ & PM & $7 / 26 / 00$ & 28478.005 & 3564.28 \\
\hline 0280 & $2: 23: 06$ & PM & $7 / 26 / 00$ & 28300.061 & 4105.71 \\
\hline 281 & $2: 23: 08$ & PM & $7 / 26 / 00$ & 32823.695 & 3701.63 \\
\hline 282 & $2: 23: 09$ & PM & $7 / 26 / 00$ & 33387.923 & 4038.13 \\
\hline 283 & $2: 23: 11$ & PM & $7 / 26 / 00$ & 29355.975 & 4069.06 \\
\hline 284 & $2: 23: 12$ & PM & $7 / 26 / 00$ & 28382.937 & 3342.14 \\
\hline 0285 & $2: 23: 14$ & PM & $7 / 26 / 00$ & 30724.558 & 3885.39 \\
\hline 0286 & $2: 23: 15$ & PM & $7 / 26 / 00$ & 27757.153 & 3930.70 \\
\hline 0287 & $2: 23: 17$ & PM & $7 / 26 / 00$ & 26654.744 & 3628.65 \\
\hline 288 & $2: 23: 18$ & PM & $7 / 26 / 00$ & 29990.040 & 3261.24 \\
\hline 289 & $2: 23: 20$ & PM & $7 / 26 / 00$ & 26266.053 & 3459.46 \\
\hline 0290 & $2: 23: 21$ & PM & $7 / 26 / 00$ & 29792.382 & 3235.47 \\
\hline 0291 & $2: 23: 23$ & PM & $7 / 26 / 00$ & 29925.545 & 3502.24 \\
\hline 0292 & $2: 23: 24$ & PM & $7 / 26 / 00$ & 30293.686 & 3418.37 \\
\hline 293 & $2: 23: 26$ & PM & $7 / 26 / 00$ & 26825.396 & 4055.88 \\
\hline 294 & $2: 23: 27$ & PM & $7 / 26 / 00$ & 29884.793 & 3575.11 \\
\hline 0295 & $2: 23: 29$ & PM & $7 / 26 / 00$ & 28250.901 & 3292.14 \\
\hline 296 & $2: 23: 30$ & PM & $7 / 26 / 00$ & 30777.533 & 3963.72 \\
\hline 297 & $2: 23: 32$ & PM & $7 / 26 / 00$ & 29653.279 & 3900.37 \\
\hline 298 & $2: 23: 33$ & PM & $7 / 26 / 00$ & 27579.669 & 3436.39 \\
\hline 299 & $2: 23: 35$ & PM & $7 / 26 / 00$ & 30178.507 & 3094.71 \\
\hline 300 & $2: 23: 36$ & PM & $7 / 26 / 00$ & 27131.350 & 4025.15 \\
\hline 301 & $2: 23: 38$ & $\mathrm{PM}$ & $7 / 26 / 00$ & 29507.319 & 3365.78 \\
\hline 302 & $2: 23: 39$ & PM & $7 / 26 / 00$ & 28113.906 & 3247.27 \\
\hline 303 & $2: 23: 41$ & PM & $7 / 26 / 00$ & 26782.962 & 3094.40 \\
\hline 304 & $2: 23: 42$ & PM & $7 / 26 / 00$ & 27328.093 & 3513.01 \\
\hline 305 & $2: 23: 44$ & PM & $7 / 26 / 00$ & 26860.833 & 3711.70 \\
\hline 306 & $2: 23: 45$ & $\mathrm{PM}$ & $7 / 26 / 00$ & 26112.717 & 3287.34 \\
\hline 307 & $2: 23: 47$ & PM & $7 / 26 / 00$ & 21395.226 & 4076.16 \\
\hline 0308 & $2: 23: 48$ & PM & $7 / 26 / 00$ & 31272.902 & 3859.81 \\
\hline 309 & $2: 23: 50$ & PM & $7 / 26 / 00$ & 24051.959 & 3337.18 \\
\hline 310 & $2: 23: 51$ & PM & $7 / 26 / 00$ & 25725.613 & 3330.59 \\
\hline 311 & $2: 23: 53$ & $\mathrm{PM}$ & $7 / 26 / 00$ & 27228.003 & 3652.81 \\
\hline 312 & $2: 23: 54$ & PM & $7 / 26 / 00$ & 27745.228 & 3113.10 \\
\hline 0313 & $2: 23: 56$ & PM & $7 / 26 / 00$ & 27373.109 & 4399.35 \\
\hline 20314 & $2: 23: 57$ & PM & $7 / 26 / 00$ & 21812.266 & 3722.86 \\
\hline C0315 & $2: 23: 59$ & PM & $7 / 26 / 00$ & 23507.422 & 3142.66 \\
\hline 316 & $2: 24: 00$ & PM & $7 / 26 / 00$ & 23778.373 & 3798.10 \\
\hline 317 & $2: 24: 02$ & PM & $7 / 26 / 00$ & 25942.109 & 3072.82 \\
\hline 318 & $2: 24: 03$ & PM & $7 / 26 / 00$ & 22442.252 & 2980.11 \\
\hline 319 & $2: 24: 05$ & PM & $7 / 26 / 00$ & 21822.560 & 3092.58 \\
\hline 0320 & $2: 24: 06$ & PM & $7 / 26 / 00$ & 18035.495 & 2912.99 \\
\hline 321 & $2: 24: 08$ & PM & $7 / 26 / 00$ & 25489.463 & 3736.60 \\
\hline 322 & $2: 24: 09$ & PM & $7 / 26 / 00$ & 22873.700 & 3774.43 \\
\hline 323 & $2: 24: 11$ & PM & $7 / 26 / 00$ & 21416.791 & 3131.43 \\
\hline 324 & $2: 24: 12$ & PM & $7 / 26 / 00$ & 23502.534 & 3486.31 \\
\hline 325 & $2: 24: 14$ & PM & $7 / 26 / 00$ & 24115.811 & 3803.97 \\
\hline 326 & $2: 24: 15$ & PM & $7 / 26 / 00$ & 24576.760 & 3449.46 \\
\hline
\end{tabular}


C0327 2:24:17 PM 7/26/00 C0328 2:24:18 PM 7/26/00 C0329 2:24:20 PM 7/26/00 C0330 2:24:21 PM 7/26/00 C0331 2:24:23 PM 7/26/00 C0332 2:24:24 PM $7 / 26 / 00$ C0333 2:24:26 PM 7/26/00 C0334 2:24:27 PM 7/26/00 C0335 2:24:29 PM 7/26/00 C0336 2:24:30 PM $7 / 26 / 00$ C0337 2:24:32 PM $7 / 26 / 00$ C0338 2:24:33 PM 7/26/00 C0339 2:24:35 PM 7/26/00 C0340 2:24:36 PM 7/26/00 C0341 2:24:38 PM 7/26/00 C0342 2:24:39 PM 7/26/00 C0343 2:24:41 PM 7/26/00 C0344 2:24:42 PM 7/26/00 C0345 2:24:44 PM 7/26/00 C0346 2:24:45 PM 7/26/00 C0347 2:24:47 PM 7/26/00 C0348 2:24:48 PM 7/26/00 C0349 2:24:50 PM 7/26/00 C0350 2:24:51 PM 7/26/00 C0351 2:24:53 PM 7/26/00 C0352 2:24:54 PM 7/26/00 C0353 2:24:56 PM 7/26/00 C0354 2:24:57 PM 7/26/00 C0355 2:24:58 PM 7/26/00 C0356 2:25:00 PM 7/26/00 C0357 2:25:02 PM 7/26/00 C0358 2:25:03 PM 7/26/00 C0359 2:25:04 PM 7/26/00 C0360 2:25:06 PM 7/26/00 C0361 2:25:07 PM 7/26/00 C0362 2:25:09 PM 7/26/00 C0363 2:25:11 PM 7/26/00 C0364 2:25:12 PM 7/26/00 C0365 2:25:13 PM 7/26/00 C0366 2:25:15 PM 7/26/00 C0367 2:25:16 PM 7/26/00 C0368 2:25:18 PM 7/26/00 C0369 2:25:19 PM 7/26/00 C0370 2:25:21 PM 7/26/00 C0371 2:25:22 PM 7/26/00 C0372 2:25:24 PM 7/26/00 C0373 2:25:26 PM 7/26/00 C0374 2:25:27 PM 7/26/00 C0375 2:25:28 PM 7/26/00 C0376 2:25:30 PM 7/26/00 C0377 2:25:31 PM 7/26/00 C0378 2:25:33 PM 7/26/00 C0379 2:25:34 PM $7 / 26 / 00$ C0380 2:25:36 PM $7 / 26 / 00$ C0381 $2: 25: 37$ PM $7 / 26 / 00$ C0382 2:25:39 PM 7/26/00

$\begin{array}{ll}23888.621 & 3175.93 \\ 24000.169 & 3598.02 \\ 24567.090 & 2929.50 \\ 25748.959 & 2916.39 \\ 23310.981 & 3091.68 \\ 22802.783 & 2698.84 \\ 22879.992 & 2794.63 \\ 21162.784 & 2946.51 \\ 24048.325 & 3646.58 \\ 24313.494 & 3005.26 \\ 25619.977 & 3654.70 \\ 26966.153 & 3049.35 \\ 25932.460 & 3518.13 \\ 25394.488 & 3868.30 \\ 24978.652 & 3482.48 \\ 20440.158 & 2804.54 \\ 21886.004 & 3764.29 \\ 24246.953 & 2925.65 \\ 21099.011 & 3283.58 \\ 23150.609 & 2807.46 \\ 22958.947 & 2762.59 \\ 23018.359 & 2992.89 \\ 21419.499 & 3500.46 \\ 27343.861 & 3640.12 \\ 23912.667 & 3424.29 \\ 24836.896 & 3419.49 \\ 26569.596 & 3157.41 \\ 25064.933 & 3640.00 \\ 20635.182 & 3773.69 \\ 20538.947 & 3179.46 \\ 25076.962 & 3587.41 \\ 23602.759 & 3020.96 \\ 21612.731 & 3427.49 \\ 24007.445 & 3349.98 \\ 24302.872 & 3939.99 \\ 22236.476 & 2231.78 \\ 24636.008 & 3471.28 \\ 20822.600 & 3014.64 \\ 21019.397 & 2851.44 \\ 22433.364 & 3111.93 \\ 22064.208 & 3249.69 \\ 21084.077 & 2882.38 \\ 21893.208 & 3179.37 \\ 27672.114 & 3801.80 \\ 26189.412 & 3475.45 \\ 22717.831 & 2876.32 \\ 25578.850 & 2649.80 \\ 20088.712 & 3678.27 \\ 17014.007 & 2788.90 \\ 23888.718 & 3705.79 \\ 20986.472 & 3570.82 \\ 21909.566 & 3716.36 \\ 24098.067 & 3847.28 \\ 26538.645 & 3827.31 \\ 17865.829 & 3434.50 \\ 24256.942 & 3078.79\end{array}$




$\begin{array}{ll}24758.229 & 3109.65 \\ 21157.056 & 3708.63 \\ 21189.982 & 3623.08 \\ 20237.721 & 3808.42 \\ 22054.143 & 3668.96 \\ 21247.605 & 3523.73 \\ 24010.442 & 4215.80 \\ 22697.261 & 3250.56 \\ 23696.826 & 2852.43 \\ 23055.587 & 3949.45 \\ 20172.215 & 3660.05 \\ 23269.555 & 3464.92 \\ 22356.238 & 3332.22 \\ 25455.447 & 2979.81 \\ 21675.170 & 3124.18 \\ 22449.263 & 3239.85 \\ 20228.608 & 3260.80 \\ 20907.140 & 3409.71\end{array}$

Revision 0 
B-2

Data to be used in test case 5 .

\begin{tabular}{|c|c|c|c|c|}
\hline \multirow{2}{*}{\multicolumn{5}{|c|}{$\begin{array}{l}\text { LANL/Hanford Method } \\
\text { Water Errort }\end{array}$}} \\
\hline & & & & \\
\hline $\mathrm{C} 0001$ & $7 / 26 / 00$ & $14: 16: 07$ & 16 & 15 \\
\hline $\mathrm{C} 0002$ & $7 / 26 / 00$ & $14: 16: 08$ & 24 & 71 \\
\hline $\mathrm{C} 0003$ & $7 / 26 / 00$ & $14: 16: 10$ & 0 & 79 \\
\hline $\mathrm{C} 0004$ & $7 / 26 / 00$ & $14: 16: 11$ & 0 & 79 \\
\hline C0005 & $7 / 26 / 00$ & $14: 16: 13$ & 22 & 17 \\
\hline C0006 & $7 / 26 / 00$ & $14: 16: 14$ & 18 & \\
\hline $\mathrm{C} 0007$ & $7 / 26 / 00$ & $14: 16: 16$ & 460.968 & 2576.06 \\
\hline C0008 & $7 / 26 / 00$ & $14: 16: 17$ & 20 & 02 \\
\hline C0009 & $7 / 26 / 00$ & $14: 16: 19$ & 5945.19 & 3057.07 \\
\hline C0010 & $7 / 26 / 00$ & $14: 16: 21$ & 23 & 36 \\
\hline $\mathrm{C} 0011$ & $7 / 26 / 00$ & $14: 16: 22$ & 4079.064 & 2439.07 \\
\hline $\mathrm{C} 0012$ & $7 / 26 / 00$ & $14: 16: 23$ & 25 & 78 \\
\hline $\mathrm{C} 0013$ & $7 / 26 / 00$ & $14: 16: 25$ & 25 & $80^{\circ}$ \\
\hline $\mathrm{C} 0014$ & $7 / 26 / 00$ & $14: 16: 26$ & 20 & 27 \\
\hline $\mathrm{C} 0015$ & $7 / 26 / 00$ & $14: 16: 28$ & 3717.884 & 2625.95 \\
\hline $\mathrm{C} 0016$ & $7 / 26 / 00$ & $14: 16: 29$ & 25 & 67 \\
\hline $\mathrm{C} 0017$ & $7 / 26 / 00$ & $14: 16: 31$ & 23 & 97 \\
\hline $\mathrm{C} 0018$ & $7 / 26 / 00$ & $14: 16: 32$ & 23 & 43 \\
\hline Co019 & $7 / 26 / 00$ & $14: 16: 34$ & 7523.436 & 2616.44 \\
\hline $\mathrm{C} 0020$ & $7 / 26 / 00$ & $14: 16: 35$ & 1640.644 & 3041.07 \\
\hline $\mathrm{C} 0021$ & $7 / 26 / 00$ & $14: 16: 37$ & 21 & 77 \\
\hline $\mathrm{C} 0022$ & $7 / 26 / 00$ & $14: 16: 38$ & 22 & \\
\hline $\mathrm{C} 0023$ & $7 / 26 / 00$ & $14: 16: 40$ & 2068.038 & 2091.7 \\
\hline $\mathrm{COO} 24$ & $7 / 26 / 00$ & $14: 16: 41$ & 21 & 16 \\
\hline $\mathrm{C} 0025$ & $7 / 26 / 00$ & $14: 16: 43$ & 6930.988 & 2594.54 \\
\hline $\mathrm{C} 0026$ & $7 / 26 / 00$ & $14: 16: 44$ & 22 & 84 \\
\hline $\mathrm{C} 0027$ & $7 / 26 / 00$ & $14: 16: 46$ & 22 & 01 \\
\hline $\mathrm{C} 0028$ & $7 / 26 / 00$ & $14: 16: 47$ & 19 & 38 \\
\hline C0029 & $7 / 26 / 00$ & $14: 16: 49$ & 0 & 22 \\
\hline 00030 & $7 / 26 / 00$ & $14: 16: 50$ & 14.002 & 2798.82 \\
\hline $\cos 31$ & $7 / 26 / 00$ & $14: 16: 52$ & 1627.23 & 2573.27 \\
\hline 00032 & $7 / 26 / 00$ & $14: 16: 53$ & 3188.682 & 2522.79 \\
\hline $\mathrm{COO} 33$ & $7 / 26 / 00$ & $14: 16: 55$ & 1236.862 & 2037.37 \\
\hline $\mathrm{C} 0034$ & $7 / 26 / 00$ & $14: 16: 56$ & 27 & 37 \\
\hline C0035 & $7 / 26 / 00$ & $14: 16: 58$ & 22 & \\
\hline $\mathrm{C} 0036$ & $7 / 26 / 00$ & $14: 16: 59$ & 24 & 81 \\
\hline $\mathrm{C} 0037$ & $7 / 26 / 00$ & $14: 17: 01$ & 23 & 75 \\
\hline C0038 & $7 / 26 / 00$ & $14: 17: 02$ & 8267.234 & 2310.7 \\
\hline C0039 & $7 / 26 / 00$ & $14: 17: 04$ & 1148.746 & 2381.76 \\
\hline $\mathrm{COO} 40$ & $7 / 26 / 00$ & $14: 17: 05$ & 4144.21 & 2824.6 \\
\hline C0041 & $7 / 26 / 00$ & $14: 17: 07$ & 29 & 98 \\
\hline $\mathrm{COO42}$ & $7 / 26 / 00$ & $14: 17: 08$ & 23 & 09 \\
\hline $\mathrm{C} 0043$ & $7 / 26 / 00$ & $14: 17: 10$ & 1555.672 & 2116.64 \\
\hline $\mathrm{COO} 44$ & $7 / 26 / 00$ & $14: 17: 11$ & 0 & 95 \\
\hline C0045 & $7 / 26 / 00$ & $14: 17: 13$ & 25 & 59 \\
\hline $\mathrm{C} 0046$ & $7 / 26 / 00$ & $14: 17: 14$ & 37 & 91 \\
\hline $\mathrm{C} 0047$ & $7 / 26 / 00$ & $14: 17: 16$ & 25 & 04 \\
\hline $\mathrm{C} 0048$ & $7 / 26 / 00$ & $14: 17: 17$ & 22 & 56 \\
\hline
\end{tabular}




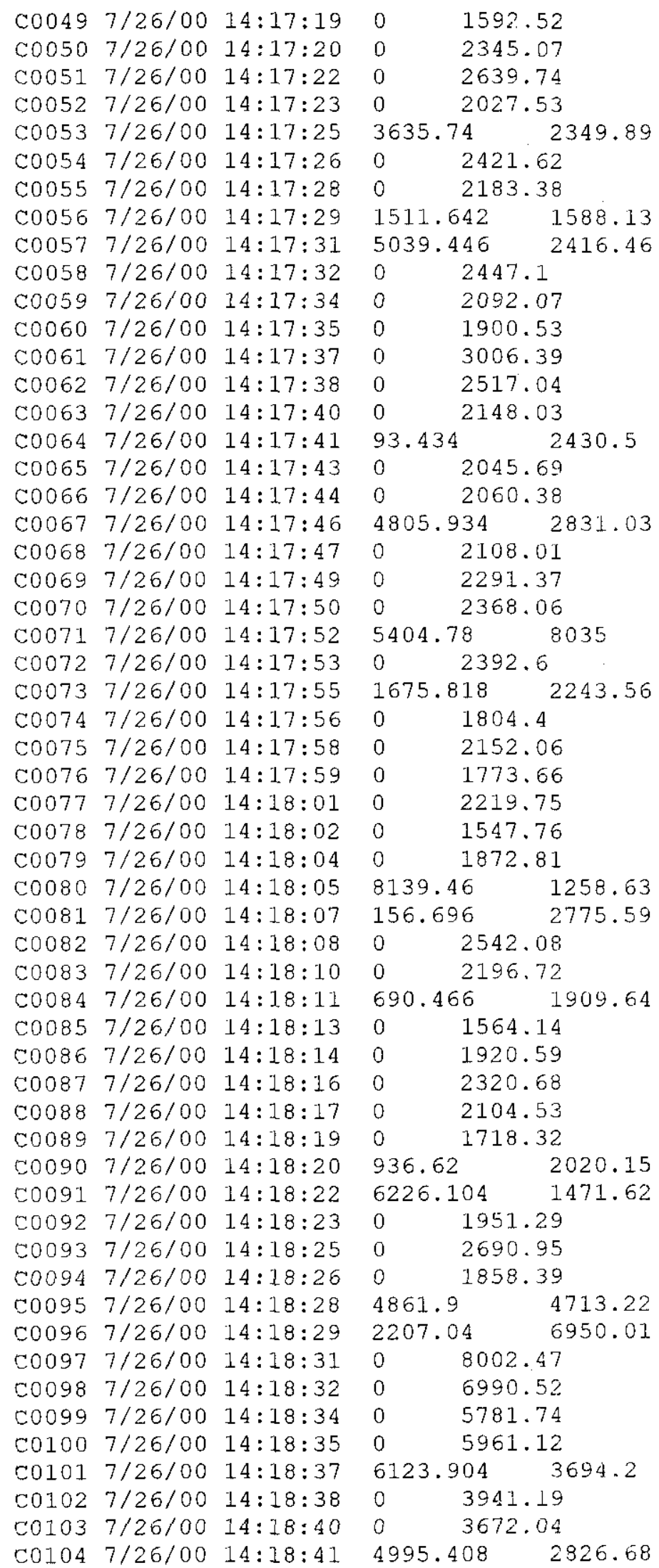


C0105 7/26/00 14:18:43 C0106 7/26/00 14:18:44 C0107 7/26/00 14:18:46 C0108 7/26/00 14:18:47 C0109 7/26/00 14:18:50 C0110 7/26/00 14:18:52 C0111 7/26/00 14:18:53 C0112 7/26/00 14:18:55 C0113 7/26/00 14:18:56 C0114 7/26/00 14:18:58 C0115 7/26/00 14:18:59 C0116 7/26/00 14:19:01 C0117 7/26/00 14:19:02 C0118 7/26/00 14:19:04 C0119 7/26/00 14:19:05 C0120 7/26/00 14:19:07 C0121 7/26/00 14:19:08 C0122 7/26/00 14:19:10 C0123 7/26/00 14:19:11 C0124 7/26/00 14:19:13 C0125 7/26/00 14:19:14 C0126 7/26/00 14:19:16 C0127 7/26/00 14:19:17 C0128 7/26/00 14:19:19 C0129 7/26/00 14:19:20 C0130 7/26/00 14:19:22 C0131 7/26/00 14:19:23 C0132 7/26/00 14:19:25 C0133 7/26/00 14:19:26 C0134 7/26/00 14:19:28 C0135 7/26/00 14:19:29 C0136 7/26/00 14:19:31 $\mathrm{C} 0137$ 7/26/00 14:19:32 C0138 7/26/00 14:19:33 C0139 7/26/00 14:19:35 C0140 7/26/00 14:19:36 C0141 7/26/00 14:19:38 C0142 7/26/00 14:19:39 $\mathrm{C} 0143$ 7/26/00 14:19:41 C0144 7/26/00 14:19:42 C0145 7/26/00 14:19:44 C0146 7/26/00 14:19:45 $00147 \quad 7 / 26 / 00 \quad 14: 19: 47$ C0148 7/26/00 14:19:48 C0149 7/26/00 14:19:50 C0150 7/26/00 14:19:51 C0151 7/26/00 14:19:53 C0152 7/26/00 14:19:54 C0153 7/26/00 14:19:56 C0154 7/26/00 14:19:57 c0155 7/26/00 14:19:59 C0156 7/26/00 14:20:00 C0157 7/26/00 14:20:02 C0158 7/26/00 14:20:03 C0159 7/26/00 14:20:05 C0160 7/26/00 14:20:06
$0 \quad 1519.57$

$92.826 \quad 2840.6$

$0 \quad 2192.68$

$622.224 \quad 1868.83$

$48768.014 \quad 2647.81$

$37562.7 \quad 2936.09$

$38243.334 \quad 3961.02$

$42796.968 \quad 3448.65$

$38822.344 \quad 2899.09$

$37682.83 \quad 2793.44$

$33658.984 \quad 2906.88$

$35757.116 \quad 3657.23$

$33500.026 \quad 2896.06$

$32855.928 \quad 2924.8$

$30457.438 \quad 3452.61$

$36599.176 \quad 2902.45$

$35740.03 \quad 2532.09$

$41102.224 \quad 2927.84$

$46791.764 \quad 3979.4$

$58213.4 \quad 3505.07$

$55956.334 \quad 3145.46$

$52627.404 \quad 2985.4$

$61564.904 \quad 4037.43$

$68288.182 \quad 3839.62$

$55998.842 \quad 3754.3$

$55762.582 \quad 2844.22$

$58914.03 \quad 3629.77$

$49995.606 \quad 3382.96$

$56949.96 \quad 3787.61$

$55892.85 \quad 2992.75$

$57898.95 \quad 3699.72$

$66359.372 \quad 3648.46$

$56764.382 \quad 4176.71$

$61636.094 \quad 4110.75$

$63294.206 \quad 3999.12$

$57811.658 \quad 3870.79$

$50913.59 \quad 3447.89$

$58681.016 \quad 4141.14$

$58050.962 \quad 4141.64$

$49754.698 \quad 2802.05$

$57248.544 \quad 3714.42$

$47377.404 \quad 3965.29$

$50974.924 \quad 3545.44$

$52131.31 \quad 4074.37$

$45056.46 \quad 3313.4$

$43265.208 \quad 3555.7$

$55205.128 \quad 3421.6$

$53848.612 \quad 3265.41$

$56327.326 \quad 4689.83$

$40460.86 \quad 3372.25$

$52793.146 \quad 2805.49$

$53010.594 \quad 3332.41$

$49461.476 \quad 3850.73$

$60621.496 \quad 3721.6$

$55024.79 \quad 3546.45$

$42584.474 \quad 3142.44$

Revision 0 


\begin{tabular}{|c|c|c|c|c|}
\hline 161 & & $14: 20: 0$ & & \\
\hline C0162 & $7 / 26 / 00$ & $14: 20: 09$ & 57452.992 & 3375.04 \\
\hline 163 & $7 / 26 / 00$ & $14: 20: 11$ & 53325.838 & 3670.3 \\
\hline 164 & $7 / 26 / 00$ & $14: 20: 12$ & 64444.196 & 4153.4 \\
\hline 165 & $7 / 26 / 00$ & $14: 20: 14$ & 57054.07 & 3487.87 \\
\hline 166 & $7 / 26 / 00$ & $14: 20: 15$ & 55206.602 & 4222.3 \\
\hline 167 & $7 / 26 / 00$ & $14: 20: 17$ & 53582.822 & 3678.28 \\
\hline 168 & $7 / 26 / 00$ & $14: 20: 18$ & 45843.092 & 4000.47 \\
\hline 169 & $7 / 26 / 00$ & $14: 20: 20$ & 53586.484 & 3615.21 \\
\hline 170 & $7 / 26 / 00$ & $14: 20: 21$ & 53599.008 & 4153.96 \\
\hline 171 & $7 / 26 / 00$ & $14: 20: 23$ & 56358.178 & 3948.62 \\
\hline 172 & $7 / 26 / 00$ & $14: 20: 24$ & 51761 & 3.5 \\
\hline 173 & $7 / 26 / 00$ & $14: 20: 26$ & 53068.2 & 3552 \\
\hline 174 & $7 / 26 / 00$ & $14: 20: 27$ & 48709.54 & 3221.18 \\
\hline 175 & $7 / 26 / 00$ & $14: 20: 29$ & 53411.55 & 3409.3 \\
\hline 176 & $7 / 26 / 00$ & $14: 20: 30$ & 56399.194 & 2983.12 \\
\hline 177 & $7 / 26 / 00$ & $14: 20: 32$ & 63953.63 & 4144.3 \\
\hline 178 & $7 / 26 / 00$ & $14: 20: 33$ & 53071.622 & 3596.24 \\
\hline 179 & $7 / 26 / 00$ & $14: 20: 35$ & 48404.28 & 3254.32 \\
\hline 80 & $7 / 26 / 00$ & $14: 20: 36$ & 50077.924 & 2945.74 \\
\hline & $7 / 26 / 00$ & $14: 20: 38$ & 54027.998 & 2953.62 \\
\hline 2 & $7 / 26 / 00$ & $14: 20: 39$ & 51483.144 & 3077.23 \\
\hline 183 & $7 / 26 / 00$ & $14: 20: 41$ & 44475.534 & 3887.23 \\
\hline 84 & $7 / 26 / 00$ & $14: 20: 42$ & 53102.048 & 3727.47 \\
\hline 85 & $7 / 26 / 00$ & $14: 20: 44$ & 46466.688 & 3289.86 \\
\hline & $7 / 26 / 00$ & $14: 20: 45$ & 56729.6 & 3257.36 \\
\hline 187 & $7 / 26 / 00$ & $14: 20: 47$ & 52729.272 & 3839.27 \\
\hline L88 & $7 / 26 / 00$ & $14: 20: 48$ & 54925.562 & 3380.21 \\
\hline 189 & $7 / 26 / 00$ & $14: 20: 50$ & 57266.858 & 3413.47 \\
\hline 0 & $7 / 26 / 00$ & $14: 20: 51$ & 53178.48 & 3017.29 \\
\hline & $7 / 26 / 00$ & $14: 20: 53$ & 48162.874 & 0.86 \\
\hline 92 & $7 / 26 / 00$ & $14: 20: 54$ & 55151.922 & 3906.04 \\
\hline 193 & $7 / 26 / 00$ & $14: 20: 56$ & 50891.298 & 3028.1 \\
\hline 194 & $7 / 26 / 00$ & $14: 20: 57$ & 47799.914 & 2963.55 \\
\hline 195 & $7 / 26 / 00$ & $14: 20: 59$ & 51050.734 & 3253.78 \\
\hline 6 & $7 / 26 / 00$ & $14: 21: 00$ & 3.686 & 52.16 \\
\hline & $7 / 26 / 00$ & $14: 21: 02$ & 51764.608 & 3470.53 \\
\hline 98 & $7 / 26 / 00$ & $14: 21: 03$ & 54567.9 & 3931.53 \\
\hline-99 & $7 / 26 / 00$ & $14: 21: 05$ & 60468.518 & 3245.29 \\
\hline 100 & $7 / 26 / 00$ & $14: 21: 06$ & 55865.304 & 3658.87 \\
\hline 1 & $7 / 26 / 00$ & $14: 21: 08$ & 56643.968 & 3356.5 \\
\hline & $7 / 26 / 00$ & $14: 21: 09$ & 3.596 & 4245.77 \\
\hline 03 & $7 / 26 / 00$ & $14: 21: 11$ & 59298.212 & 3598.41 \\
\hline 204 & $7 / 26 / 00$ & $14: 21: 12$ & 62013.996 & 3859.19 \\
\hline 05 & $7 / 26 / 00$ & $14: 21: 14$ & 62119.214 & 3606.27 \\
\hline 6 & $7 / 26 / 00$ & $14: 21: 15$ & 52789.826 & 4077.87 \\
\hline 07 & $7 / 26 / 00$ & $14: 21: 17$ & 61149.602 & 3545.75 \\
\hline 208 & $7 / 26 / 00$ & $14: 21: 18$ & 56064.9 & 3674.16 \\
\hline 209 & $7 / 26 / 00$ & $14: 21: 20$ & 51786.974 & 3390.7 \\
\hline & $7 / 26 / 00$ & $14: 21: 21$ & $65484.00 E$ & 4145.9 \\
\hline & $7 / 26 / 00$ & $14: 21: 23$ & 67476.032 & 3924.32 \\
\hline & $7 / 26 / 00$ & $14: 21: 24$ & 60708.172 & 4137.44 \\
\hline & $7 / 26 / 00$ & $14: 21: 26$ & 65300.138 & 4329.52 \\
\hline & $7 / 26 / 00$ & $14: 21: 27$ & 60875.486 & 3957.82 \\
\hline & $7 / 26 / 00$ & $14: 21: 29$ & 63295.236 & 3812.46 \\
\hline & $7 / 26 / 00$ & $14: 21: 30$ & 58525.986 & 3346.66 \\
\hline
\end{tabular}




\begin{tabular}{|c|c|c|c|c|}
\hline 217 & $7 / 2$ & & & \\
\hline 218 & $7 / 26 / 00$ & $14: 21: 33$ & 67401.242 & 29.34 \\
\hline 19 & $7 / 26 / 00$ & $14: 21: 35$ & 60719.472 & 03.23 \\
\hline 20 & $7 / 26 / 00$ & $14: 21: 36$ & 62981.858 & \\
\hline 221 & $7 / 26 / 00$ & $14: 21: 38$ & 67582.966 & $3625 \quad 48$ \\
\hline 22 & $7 / 26 / 00$ & $14: 21: 39$ & 69149.632 & 367317 \\
\hline 223 & $7 / 26 / 00$ & $14: 21: 41$ & 67059.75 & 3863.71 \\
\hline 224 & $7 / 26 / 00$ & $14: 21: 42$ & 65157.354 & 3220.9 \\
\hline 225 & $7 / 26 / 00$ & $14: 21: 44$ & 71260.022 & 3849.82 \\
\hline 226 & $7 / 26 / 00$ & $14: 21: 45$ & 66426.098 & 3128.27 \\
\hline 227 & $7 / 26 / 00$ & $14: 21: 47$ & 56977.72 & 3563.13 \\
\hline 28 & $7 / 26 / 00$ & $14: 21: 48$ & 68779.408 & 3025.68 \\
\hline 29 & $7 / 26 / 00$ & $14: 21: 50$ & 73718.106 & 4487.09 \\
\hline 230 & $7 / 26 / 00$ & $14: 21: 51$ & 62294.496 & 3913.35 \\
\hline 231 & $7 / 26 / 00$ & $14: 21: 53$ & 68927.686 & 3547.36 \\
\hline 32 & $7 / 26 / 00$ & $14: 21: 54$ & 58966.116 & \\
\hline 33 & $7 / 26 / 00$ & $14: 21: 56$ & 66881.436 & 3668.8 \\
\hline 234 & $7 / 26 / 00$ & $14: 21: 57$ & 63888.25 & 3485.3 \\
\hline 235 & $7 / 26 / 00$ & $14: 21: 59$ & 71217.136 & 3578.17 \\
\hline 236 & $7 / 26 / 00$ & $14: 22: 00$ & 68639.226 & 3651.03 \\
\hline 237 & $7 / 26 / 00$ & $14: 22: 02$ & 65491.062 & 3622.45 \\
\hline 238 & $7 / 26 / 00$ & $14: 22: 03$ & 72998.478 & 4282.63 \\
\hline 239 & $7 / 26 / 00$ & $14: 22: 05$ & 73277.722 & 4121.03 \\
\hline 240 & $7 / 26 / 00$ & $14: 22: 06$ & 65797.078 & 3644.89 \\
\hline 41 & $7 / 26 / 00$ & $14: 22: 08$ & 71755.01 & 3790.11 \\
\hline 242 & $7 / 26 / 00$ & $14: 22: 09$ & 74692.906 & 4358.8 \\
\hline 43 & $7 / 26 / 00$ & $14: 22: 11$ & 69409.734 & 4752.45 \\
\hline 244 & $7 / 26 / 00$ & $14: 22: 12$ & 69375.034 & 3990.07 \\
\hline 5 & $7 / 26 / 00$ & $14: 22: 14$ & 5.41 & 3422.15 \\
\hline 46 & $7 / 26 / 00$ & $14: 22: 15$ & 64968.678 & 86 \\
\hline 247 & $7 / 26 / 00$ & $14: 22: 17$ & 69298.87 & 3766.01 \\
\hline 248 & $7 / 26 / 00$ & $14: 22: 18$ & 70402.364 & 3799.17 \\
\hline 249 & $7 / 26 / 00$ & $14: 22: 20$ & 70957.326 & 4709.13 \\
\hline 50 & $7 / 26 / 00$ & $14: 22: 21$ & 73771.634 & 3709.44 \\
\hline 1 & $7 / 26 / 00$ & $22: 23$ & 9.032 & 3266.8 \\
\hline 252 & $7 / 26 / 00$ & $14: 22: 24$ & 73649.12 & 3869.56 \\
\hline 253 & $7 / 26 / 00$ & $14: 22: 26$ & 65749.212 & 3214.94 \\
\hline 254 & $7 / 26 / 00$ & $14: 22: 27$ & 71686.726 & 3856.96 \\
\hline 55 & $7 / 26 / 00$ & $14: 22: 29$ & 64770.498 & .07 \\
\hline 56 & $7 / 2$ & $2: 30$ & 6.912 & 3809.25 \\
\hline 57 & $7 / 26 / 00$ & $14: 22: 32$ & 62690.072 & 4248.21 \\
\hline 258 & $7 / 26 / 00$ & $14: 22: 33$ & 71702.306 & 3776.29 \\
\hline 59 & $7 / 26 / 00$ & $14: 22: 35$ & 60645.154 & 3136.91 \\
\hline 50 & $7 / 26 / 00$ & $14: 22: 36$ & 62449.392 & 4.12 \\
\hline 61 & $7 / 26 / 00$ & $14: 22: 38$ & 65768.75 & 3156.62 \\
\hline 62 & $7 / 26 / 00$ & $14: 22: 39$ & 65904.432 & 3821.48 \\
\hline 263 & $7 / 26 / 00$ & $14: 22: 41$ & 70091.274 & 3677.75 \\
\hline 264 & $7 / 26 / 00$ & $14: 22: 42$ & 65429.318 & 3747.65 \\
\hline 65 & $7 / 26 / 00$ & $14: 22: 44$ & 67704.754 & 4547.34 \\
\hline 266 & $7 / 26 / 00$ & $14: 22: 45$ & 62591.756 & 4183.04 \\
\hline 267 & $7 / 26 / 00$ & $14: 22: 47$ & 61802.9 & 3988.77 \\
\hline & $7 / 26 / 00$ & $14: 22: 48$ & 60569.044 & 2974.95 \\
\hline & $7 / 26 / 00$ & $14: 22: 50$ & 63429.516 & 4609.81 \\
\hline & $7 / 26 / 00$ & $14: 22: 51$ & 60011.544 & 3695.73 \\
\hline & $7 / 26 / 00$ & $14: 22: 53$ & 63680.646 & 3683.31 \\
\hline & $7 / 26 / 00$ & $14: 22: 54$ & 63936.284 & 3924.58 \\
\hline
\end{tabular}




\begin{tabular}{|c|c|c|c|c|}
\hline 27 & & & 568 & 3869.81 \\
\hline 74 & 100 & $14: 22: 57$ & 64396.894 & 4250.69 \\
\hline & $6 / 00$ & $14: 22: 59$ & 49874.628 & 3731.8 \\
\hline 27 & $/ 26 / 00$ & $14: 23: 00$ & 58048.86 & 3458.79 \\
\hline 277 & $7 / 26 / 00$ & $14: 23: 02$ & 51164.002 & 3874.96 \\
\hline 78 & $/ 26 / 00$ & $14: 23: 03$ & 54807.668 & 3507.06 \\
\hline & $/ 26 / 00$ & $14: 23: 05$ & 56956.01 & 3564.28 \\
\hline 28 & $/ 26 / 00$ & $14: 23: 06$ & 56600.122 & 4105.71 \\
\hline 28 & $/ 26 / 00$ & $14: 23: 08$ & 65647.39 & 3701.63 \\
\hline 28 & $7 / 26 / 00$ & $14: 23: 09$ & 66775.846 & 4038.13 \\
\hline 283 & $7 / 26 / 00$ & $14: 23: 11$ & 58711.95 & 4069.06 \\
\hline & $/ 26 / 00$ & $14: 23: 12$ & 56765.874 & 3342.14 \\
\hline & $/ 26 / 00$ & $14: 23: 14$ & 61449.116 & 3885.39 \\
\hline 286 & $7 / 26 / 00$ & $14: 23: 15$ & 55514.306 & 3930.7 \\
\hline 287 & $7 / 26 / 00$ & $14: 23: 17$ & 53309.488 & 3628.65 \\
\hline 288 & $7 / 26 / 00$ & $14: 23: 18$ & 59980.08 & 3251.24 \\
\hline 289 & $7 / 26 / 00$ & $14: 23: 20$ & 52532.106 & 3459.46 \\
\hline 90 & $7 / 26 / 00$ & $14: 23: 21$ & 59584.764 & 3235.47 \\
\hline 91 & $7 / 26 / 00$ & $14: 23: 23$ & 59851.09 & 3502.24 \\
\hline & $7 / 26 / 00$ & $14: 23: 24$ & 60587.372 & 3418.37 \\
\hline & $7 / 26 / 00$ & $14: 23: 26$ & 53650.792 & 4055.88 \\
\hline 4 & $7 / 26 / 00$ & $14: 23: 27$ & 59769.586 & 3575.11 \\
\hline 5 & $7 / 26 / 00$ & $14: 23: 29$ & 56501.802 & 3292.14 \\
\hline & $7 / 26 / 00$ & $14: 23: 30$ & 61555.066 & 3963.72 \\
\hline 91 & $7 / 26 / 00$ & $14: 23: 32$ & 59306.558 & 3900.37 \\
\hline & $7 / 26 / 00$ & $14: 23: 33$ & 55159.338 & 3436.39 \\
\hline & $7 / 26 / 00$ & $14: 23: 35$ & 60357.014 & \\
\hline & $7 / 26 / 00$ & $14: 23: 36$ & 54262.7 & 4025.15 \\
\hline 01 & $7 / 26 / 00$ & $14: 23: 38$ & 59014.638 & 3365.78 \\
\hline 02 & $7 / 26 / 00$ & $14: 23: 39$ & 56227.812 & 3247.27 \\
\hline & $7 / 26 / 00$ & $14: 23: 41$ & 53565.924 & 3094.4 \\
\hline & $7 / 26 / 00$ & $14: 23: 42$ & 54656.186 & .01 \\
\hline & $7 / 26 / 00$ & $14: 23: 44$ & 53721.666 & 3711.7 \\
\hline & $7 / 26 / 00$ & $14: 23: 45$ & 52225.434 & 3287.34 \\
\hline 07 & $7 / 26 / 00$ & $14: 23: 47$ & 42790.452 & 4076.16 \\
\hline & $7 / 26 / 00$ & $14: 23: 48$ & 62545.804 & 3859.81 \\
\hline & $7 / 26 / 00$ & $14: 23: 50$ & 48103.918 & 3337.18 \\
\hline & $7 / 26 / 00$ & $14: 23: 51$ & 51451.226 & 3330.59 \\
\hline & $7 / 26 / 00$ & $14: 23: 53$ & 54456.006 & 3652.81 \\
\hline & $7 / 26 / 00$ & $14: 23: 54$ & 55490.456 & 3113.1 \\
\hline & $7 / 26 / 00$ & $14: 23: 56$ & 54746.218 & 4399.35 \\
\hline & $7 / 26 / 00$ & $14: 23: 57$ & 43624.532 & 3722.86 \\
\hline & $7 / 26 / 00$ & $14: 23: 59$ & 47014.844 & 3142.66 \\
\hline & $7 / 26 / 00$ & $14: 24: 00$ & 47556.746 & 3798.1 \\
\hline & $7 / 26 / 00$ & $14: 24: 02$ & 51884.218 & 3072.82 \\
\hline & $7 / 26 / 00$ & $14: 24: 03$ & 44884.504 & 2980.11 \\
\hline & $7 / 26 / 00$ & $14: 24: 05$ & 43645.12 & 3092.58 \\
\hline & $7 / 26 / 00$ & $14: 24: 06$ & 36070.99 & 2912.99 \\
\hline & $7 / 26 / 00$ & $14: 24: 08$ & 50978.926 & 3736.6 \\
\hline & $7 / 26 / 00$ & $14: 24: 09$ & 45747.4 & 3774.43 \\
\hline & $7 / 26 / 00$ & $14: 24: 11$ & 42833.582 & 3131.43 \\
\hline & $7 / 26 / 00$ & $14: 24: 12$ & 47005.068 & 3486.31 \\
\hline & $7 / 26 / 00$ & $14: 24: 14$ & 48231.622 & 3803.97 \\
\hline & $7 / 26 / 00$ & $14: 24: 15$ & 49153.52 & 3449.46 \\
\hline & $7 / 26 / 00$ & $14: 24: 17$ & 47777.242 & 3175.93 \\
\hline & $7 / 26 / 00$ & $14: 24: 18$ & 48000.338 & 3598.02 \\
\hline
\end{tabular}




\begin{tabular}{|c|c|c|c|c|}
\hline & & & & \\
\hline 330 & $7 / 26 / 00$ & $14: 24: 21$ & 51497.918 & $6.30 \quad 2$ \\
\hline 33 & $7 / 26 / 00$ & $24: 23$ & 46621.962 & \\
\hline & & $24: 24$ & 66 & .84 \\
\hline & & $24: 26$ & 759.984 & \\
\hline & & $24: 27$ & & \\
\hline 0335 & $/ 26 / 00$ & $4: 24: 29$ & 196.65 & 658 \\
\hline 0336 & $7 / 26 / 00$ & $: 24: 30$ & 48626.988 & 5.26 \\
\hline 7 & 20 & 32 & & \\
\hline & & & & $3+3$ \\
\hline & & & & \\
\hline & 0 & 36 & 76 & \\
\hline 0341 & $7 / 26 / 00$ & $4: 24: 38$ & & 2.48 \\
\hline 0342 & $7 / 26 / 00$ & $14: 24: 39$ & & \\
\hline 03 & $7 / 26 /$ & 41 & 08 & \\
\hline & 201 & $4: 24$ & & \\
\hline & & & & \\
\hline & $126 /$ & & 18 & \\
\hline 7 & $7 / 26 / 00$ & & & \\
\hline 03 & 0 & 8 & & \\
\hline & $1<25+3$ & 0 & & \\
\hline 0 & $1 / 26 /$ & 1 & & \\
\hline & $1 / 1<4$ & & & \\
\hline 2 & $7 / 26 / 00$ & & & \\
\hline & $7 / 26$ & & & $1.41+3$ \\
\hline 4 & 0 & & & \\
\hline & $7 / 2$ & 8 & & 69 \\
\hline & $7 / 26 /$ & U & & \\
\hline & $7 / 26 / 00$ & 2 & & \\
\hline $\mathrm{C}$ & $7 / 2$ & & & \\
\hline & & & & \\
\hline & 0 & & & 98 \\
\hline & $7 / 2$ & 77 & & .99 \\
\hline & $7 / 26$ & 4: & & \\
\hline & & & & .28 \\
\hline $\mathrm{CO} 3$ & $7 / 26$ & 2 & & \\
\hline & & & & \\
\hline & & & & .93 \\
\hline & $7 / 26 /$ & & 16 & .69 \\
\hline & $7 / 26 /$ & & & .38 \\
\hline & $7 / 26$ & $4: 2$ & & .37 \\
\hline & & & & \\
\hline & & & & \\
\hline & $7 / 2$ & 24 & 62 & .32 \\
\hline & $7 / 26 / 00$ & $4: 25 \quad 5$ & 7.7 & 9.8 \\
\hline $\mathrm{CO} 3$ & $7 / 26 / 00$ & $14: 25: 27$ & .424 & 8.27 \\
\hline C0375 & $7 / 26$ & 28 & & \\
\hline & & & & \\
\hline & $7 / 26$ & & 72.944 & 3570.82 \\
\hline & $7 / 26 / 00$ & 14.25 & 43819.132 & 371.6 .36 \\
\hline & $7 / 26 / 00$ & $14: 25: 34$ & 48196.134 & 3847.28 \\
\hline $\mathrm{C} 0380$ & $7 / 26 / 00$ & $14: 25: 36$ & 7.29 & 3827.31 \\
\hline & $7 / 26 / 00$ & $4: 25: 37$ & 35731.658 & 3434.5 \\
\hline & $1 / 26$ & & & 3078.79 \\
\hline & $7 / 26 / c$ & - & 516.458 & 3109.65 \\
\hline & 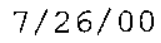 & $: 42$ & 42314.112 & 3708.6 \\
\hline
\end{tabular}


42379.964

40475.442

44108.286

42495.21

48020.884

45394.522

47393.652

46111.174

40344.43

46539.11

44712.476

50910.894

43350.34

44898.526

40457.216

41814.28
3623.08

3808.42

3668.96

3523.73

4215.8

3250.56

2852.43

3949.45

3660.05

3464.92

3332.22

2979.81

3124.18

3239.85

3260.8

3409.71

Revision 0 


\section{B-3}

Data to be used in test case 8 .

\begin{tabular}{|c|c|c|c|c|}
\hline \multirow{2}{*}{\multicolumn{4}{|c|}{ LANL/Hanford Method }} & \multirow[t]{2}{*}{ Concentrations in ppm } \\
\hline & & ter Erro. & & \\
\hline $\mathrm{C} 0001$ & $7 / 26 / 00$ & $14: 16: 07$ & 1695 & 1695.15 \\
\hline $\mathrm{C} 0002$ & $7 / 26 / 00$ & $14: 16: 08$ & 2453 & 2453.71 \\
\hline $\mathrm{C} 0003$ & $7 / 26 / 00$ & $14: 16: 10$ & 2312 & 2312.79 \\
\hline $\mathrm{C} 0004$ & $7 / 26 / 00$ & $14: 16: 11$ & 2190 & 2190.79 \\
\hline $\mathrm{C} 0005$ & $7 / 26 / 00$ & $14: 16: 13$ & 2280 & 2280.17 \\
\hline $\mathrm{C} 0006$ & $7 / 26 / 00$ & $14: 16: 14$ & 1861 & 1861.9 \\
\hline $\mathrm{C} 0007$ & $7 / 26 / 00$ & $14: 16: 16$ & 1152.42 & 2576.06 \\
\hline $\mathrm{C} 0008$ & $7 / 26 / 00$ & $14: 16: 17$ & 2023 & 2023.02 \\
\hline $\mathrm{CO009}$ & $7 / 26 / 00$ & $14: 16: 19$ & 14862.975 & 3057.07 \\
\hline $\mathrm{C} 0010$ & $7 / 26 / 00$ & $14: 16: 21$ & 2368 & 2368.36 \\
\hline $\mathrm{C} 0011$ & $7 / 26 / 00$ & $14: 16: 22$ & 10197.66 & 2439.07 \\
\hline $\mathrm{C} 0012$ & $7 / 26 / 00$ & $14: 16: 23$ & 2500 & 2500.78 \\
\hline $\mathrm{C} 0013$ & $7 / 26 / 00$ & $14: 16: 25$ & 2581 & 2581.86 \\
\hline $\mathrm{COO14}$ & $7 / 26 / 00$ & $14: 16: 26$ & 2068 & 2068.27 \\
\hline $\mathrm{COO15}$ & $7 / 26 / 00$ & $14: 16: 28$ & 9294.71 & 2625.95 \\
\hline $\mathrm{C} 0016$ & $7 / 26 / 00$ & $14: 16: 29$ & 2582 & 2582.67 \\
\hline $\mathrm{C} 0017$ & $7 / 26 / 00$ & $14: 16: 31$ & 2311 & 2311.97 \\
\hline $\mathrm{C} 0018$ & $7 / 26 / 00$ & $14: 16: 32$ & 2389 & 2389.43 \\
\hline C0019 & $7 / 26 / 00$ & $14: 16: 34$ & 18808.59 & 2616.44 \\
\hline $\mathrm{COO20}$ & $7 / 26 / 00$ & $14: 16: 35$ & 4101.61 & $61 \quad 304$ \\
\hline C0021 & $7 / 26 / 00$ & $14: 16: 37$ & 2123 & 2123.77 \\
\hline $\mathrm{C} 0022$ & $7 / 26 / 00$ & $14: 16: 38$ & 2203 & 2203.3 \\
\hline $\mathrm{C} 0023$ & $7 / 26 / 00$ & $14: 16: 40$ & 5170.095 & $209 i .7$ \\
\hline COO24 & $7 / 26 / 00$ & $14: 16: 41$ & 2157 & 2157.16 \\
\hline $\mathrm{C} 0025$ & $7 / 26 / 00$ & $14: 16: 43$ & 17327.47 & 2594.54 \\
\hline $\mathrm{C} 0026$ & $7 / 26 / 00$ & $14: 16: 44$ & $0 \quad 2269$ & 2269.84 \\
\hline $\mathrm{COO} 27$ & $7 / 26 / 00$ & $14: 16: 46$ & 2234 & \\
\hline $\mathrm{COO} 28$ & $7 / 26 / 00$ & $14: 16: 47$ & 1990 & \\
\hline C0029 & $7 / 26 / 00$ & $14: 16: 49$ & 2457 & \\
\hline C0030 & $7 / 26 / 00$ & $14: 16: 50$ & 35.005 & 2798.82 \\
\hline C0031 & $7 / 26 / 00$ & $14: 16: 52$ & 4068.075 & 2573.27 \\
\hline $\mathrm{C} 0032$ & $7 / 26 / 00$ & $14: 16: 53$ & 7971.705 & 2522.79 \\
\hline $\operatorname{co0} 33$ & $7 / 26 / 00$ & $14: 16: 55$ & 3092.155 & 2037.37 \\
\hline$C 0034$ & $7 / 26 / 00$ & $14: 16: 56$ & 2753 & \\
\hline co035 & $7 / 26 / 00$ & $14: 16: 58$ & 2258 & \\
\hline $\mathrm{C} 0036$ & $7 / 26 / 00$ & $14: 16: 59$ & 2486 & \\
\hline $\mathrm{C} 0037$ & $7 / 26 / 00$ & $14: 17: 01$ & 2398 & \\
\hline $\mathrm{C} 0038$ & $7 / 26 / 00$ & $14: 17: 02$ & 20668.085 & 2310.7 \\
\hline C0039 & $7 / 26 / 00$ & $14: 17: 04$ & 2871.865 & 2381.76 \\
\hline $\mathrm{COO40}$ & $7 / 26 / 00$ & $14: 17: 05$ & 10360.525 & 2824.6 \\
\hline $\mathrm{C} 0041$ & $7 / 26 / 00$ & $14: 17: 07$ & 2960 & \\
\hline $\mathrm{COO42}$ & $7 / 26 / 00$ & $14: 17: 08$ & 2318 & \\
\hline C0043 & $7 / 26 / 00$ & $14: 17: 10$ & 3889.18 & 2116.64 \\
\hline $\mathrm{COO44}$ & $7 / 26 / 00$ & $14: 17: 11$ & 2248 & \\
\hline C0045 & $7 / 26 / 00$ & $14: 17: 13$ & 2573 & \\
\hline C0046 & $7 / 26 / 00$ & $14: 17: 14$ & 3746 & \\
\hline $\mathrm{C} 0047$ & $7 / 26 / 00$ & $14: 17: 16$ & 2510 & \\
\hline $\mathrm{COO48}$ & $7 / 26 / 00$ & $14: 17: 17$ & 2222 & \\
\hline
\end{tabular}




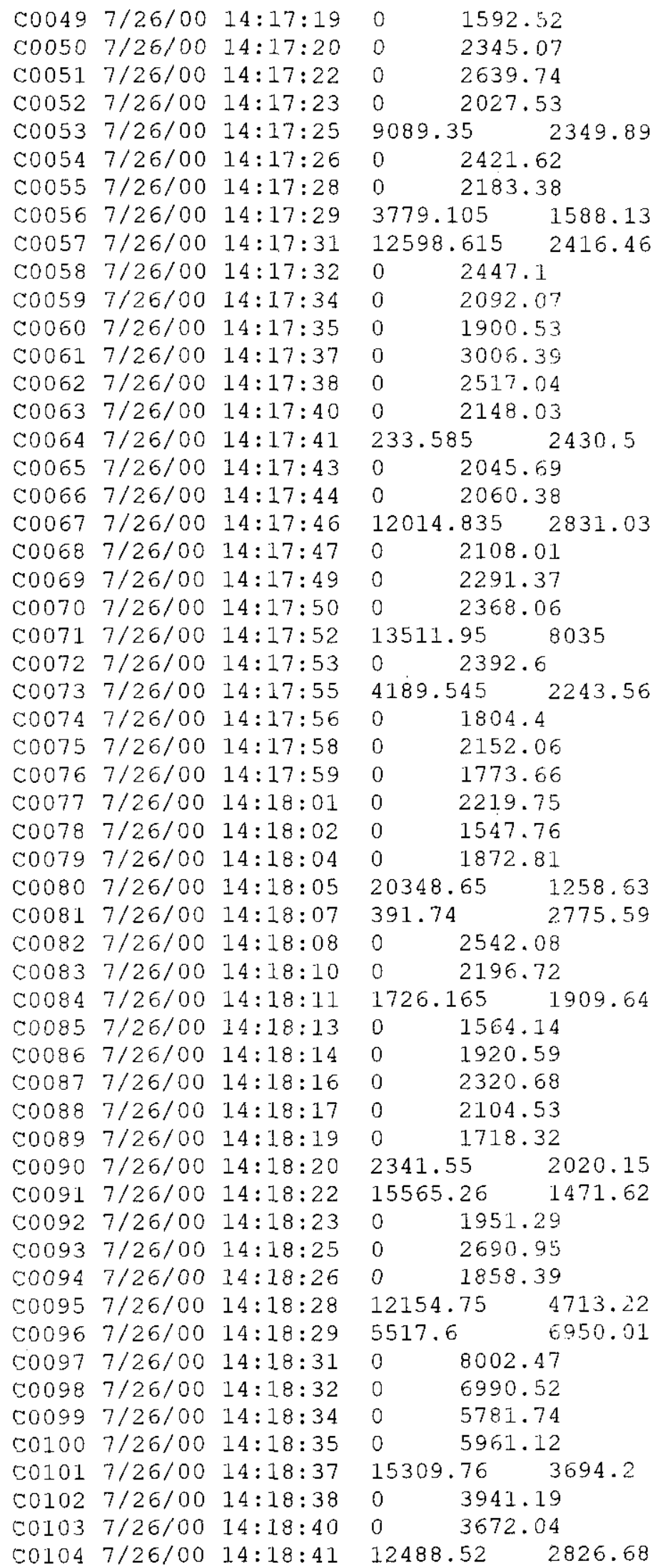




\begin{tabular}{|c|c|c|c|c|}
\hline 105 & & $14: 18: 43$ & \multicolumn{2}{|c|}{1519.57} \\
\hline & $7 / 26 / 00$ & $14: 18: 44$ & 232.065 & 2840.6 \\
\hline & 100 & $14: 18: 46$ & \multicolumn{2}{|c|}{$\begin{array}{ll}0 & 2192.68\end{array}$} \\
\hline 10 & 100 & $14: 18: 47$ & .56 & 1868.83 \\
\hline 109 & $7 / 26 / 00$ & $14: 18: 50$ & 121920.035 & 2647.81 \\
\hline 110 & $7 / 26 / 00$ & $14: 18: 52$ & 93906.75 & 2936.09 \\
\hline 1 & $7 / 26 / 00$ & $14: 18: 53$ & 95608.335 & 3961.02 \\
\hline & $7 / 26 / 00$ & $14: 18: 55$ & 106992.42 & 3448.65 \\
\hline 13 & $7 / 26 / 00$ & $14: 18: 56$ & 97055.86 & 2899.09 \\
\hline 114 & $7 / 26 / 00$ & $14: 18: 58$ & 94207.075 & 2793.44 \\
\hline 715 & $7 / 26 / 00$ & $14: 18: 59$ & 84147.46 & 2906.88 \\
\hline 10 & $7 / 26 / 00$ & $14: 19: 01$ & 89392.79 & .23 \\
\hline & $7 / 26 / 00$ & $14: 19: 02$ & 83750.065 & 2896.06 \\
\hline 18 & $7 / 26 / 00$ & $14: 19: 04$ & 82139.82 & 2924.8 \\
\hline 19 & $7 / 26 / 00$ & $14: 19: 05$ & 76143.595 & 3452.61 \\
\hline 120 & $7 / 26 / 00$ & $14: 19: 07$ & 91497.94 & .45 \\
\hline 21 & $7 / 26 / 00$ & $14: 19: 08$ & 89350.075 & \\
\hline 122 & $7 / 26 / 00$ & $14: 19: 10$ & 102755.56 & .84 \\
\hline 23 & $7 / 26 / 00$ & $14: 19: 11$ & 116979.41 & 3979.4 \\
\hline 124 & $7 / 26 / 00$ & $14: 19: 13$ & 145533.5 & 5.07 \\
\hline 125 & $7 / 26 / 00$ & $14: 19: 14$ & 139890.835 & .46 \\
\hline 26 & $7 / 26 / 00$ & $14: 19: 16$ & 131568.51 & 29 \\
\hline 27 & $7 / 26 / 00$ & $14: 19: 17$ & 153912.26 & 7.43 \\
\hline 128 & $7 / 26 / 00$ & $14: 19: 19$ & 170720.455 & 9.62 \\
\hline 129 & $7 / 26 / 00$ & $14: 19: 20$ & 139997.105 & 3754.3 \\
\hline & $7 / 26 / 00$ & $14: 19: 22$ & 139406.455 & .22 \\
\hline 31 & $7 / 26 / 00$ & $14: 19: 23$ & 147285.075 & .77 \\
\hline 32 & $7 / 26 / 00$ & $14: 19: 25$ & 124989.015 & 2.96 \\
\hline 33 & $7 / 26 / 00$ & $14: 19: 26$ & 142374.9 & .61 \\
\hline 34 & $7 / 26 / 00$ & $14: 19: 28$ & 139732.125 & 2992.75 \\
\hline & $7 / 26 / 00$ & $14: 19: 29$ & 144747.375 & .72 \\
\hline 36 & $7 / 26 / 00$ & $14: 19: 31$ & 38.43 & .46 \\
\hline 37 & $7 / 26 / 00$ & $14: 19: 32$ & 141910.955 & 5.71 \\
\hline 38 & $7 / 26 / 00$ & $14: 19: 33$ & 154090.235 & 4110.75 \\
\hline 139 & $7 / 26 / 00$ & $14: 19: 35$ & 158235.515 & 3999.12 \\
\hline & $7 / 26 / 00$ & $14: 19: 36$ & 29.145 & .79 \\
\hline 41 & $7 / 26 / 00$ & $14: 19: 38$ & 83.975 & 3447.89 \\
\hline 42 & $7 / 26 / 00$ & $14: 19: 39$ & 146702.54 & 4141.14 \\
\hline 43 & $7 / 26 / 00$ & $14: 19: 41$ & 145127.405 & 4141.64 \\
\hline 44 & $7 / 26 / 00$ & $14: 19: 42$ & 124386.745 & 2.05 \\
\hline & $7 / 26 / 00$ & $14: 19: 44$ & 21.36 & .42 \\
\hline 46 & $7 / 26 / 00$ & $14: 19: 45$ & 118443.51 & 3965.29 \\
\hline 47 & $7 / 26 / 00$ & $14: 19: 47$ & 127437.31 & 3545.44 \\
\hline 48 & $7 / 26 / 00$ & $14: 19: 48$ & 130328.275 & 4074.37 \\
\hline 49 & $7 / 26 / 00$ & $14: 19: 50$ & 112641.15 & 3313.4 \\
\hline & $7 / 26 / 00$ & $14: 19: 51$ & 108163.02 & 3555.7 \\
\hline & $7 / 26 / 00$ & $14: 19: 53$ & 138012.82 & 3421.6 \\
\hline & $7 / 26 / 00$ & $14: 19: 54$ & 134621.53 & 32.65 .41 \\
\hline & $7 / 26 / 00$ & $14: 19: 56$ & 140818.315 & 4689.83 \\
\hline 54 & $7 / 26 / 00$ & $14: 19: 57$ & 101152.15 & 3372.25 \\
\hline 55 & $7 / 26 / 00$ & $14: 19: 59$ & 131982.865 & 2805.49 \\
\hline & $7 / 26 / 00$ & $14: 20: 00$ & 132526.485 & 3332.41 \\
\hline & $7 / 26 / 00$ & $14: 20: 02$ & 123653.69 & 3850.73 \\
\hline & $7 / 26 / 00$ & $14: 20: 03$ & 151553.74 & 3721.6 \\
\hline & $7 / 26 / 00$ & $14: 20: 05$ & 137561.975 & 3546.45 \\
\hline & $7 / 26 / 00$ & $14: 20: 06$ & 106461.185 & 3142.44 \\
\hline
\end{tabular}




\begin{tabular}{|c|c|c|c|c|}
\hline 51 & 7 & 08 & 1 & \\
\hline & $7 / 26 / 00$ & $14: 20: 09$ & 143632.48 & 3375.04 \\
\hline 163 & $7 / 26 / 00$ & $14: 20: 11$ & 133314.595 & 3670.3 \\
\hline 64 & $7 / 26 / 00$ & $14: 20: 12$ & 161110.49 & 4153.46 \\
\hline 65 & 100 & $14: 20: 14$ & 142635.175 & \\
\hline 66 & 100 & $14: 20: 15$ & 138016.505 & 4222.3 \\
\hline 167 & $7 / 26 / 00$ & $14: 20: 17$ & 133957.055 & 3678.28 \\
\hline 68 & $7 / 26 / 00$ & $14: 20: 18$ & 114607.73 & \\
\hline 69 & $7 / 26 / 00$ & $14: 20: 20$ & 133966.21 & \\
\hline 170 & 100 & $14: 20: 21$ & 133997.52 & 0 \\
\hline 171 & $7 / 26 / 00$ & $14: 20: 23$ & 140895.445 & 3948.62 \\
\hline 0172 & $7 / 26 / 00$ & $14: 20: 24$ & 129404.015 & 3793.5 \\
\hline 173 & $7 / 26 / 00$ & $14: 20: 26$ & 132670.5 & 3552.78 \\
\hline 174 & $7 / 26 / 00$ & $14: 20: 27$ & 121773.85 & \\
\hline 75 & $7 / 2$ & $14: 20: 29$ & 133528.875 & 3409.3 \\
\hline 176 & $1 / 00$ & $14: 20: 30$ & 140997.985 & 2983.12 \\
\hline 0177 & $7 / 26 / 00$ & $14: 20: 32$ & 84.075 & $4 \perp 44.3$ \\
\hline 0178 & $7 / 26 / 00$ & $14: 20: 33$ & 132679.055 & .24 \\
\hline 179 & $7 / 26 / 00$ & $14: 20: 35$ & 10.7 & .32 \\
\hline 180 & $7 / 26 / 00$ & $14: 20: 36$ & 125194.81 & .74 \\
\hline & $7 / 2$ & $14: 20: 38$ & 135069.995 & 2953.62 \\
\hline 0182 & $7 / 26 / 00$ & $14: 20: 39$ & 07.86 & 3077.23 \\
\hline 0183 & $7 / 26 / 00$ & $14: 20: 41$ & 111188.835 & 3887.23 \\
\hline 184 & $7 / 26 / 00$ & $14: 20: 42$ & 5.12 & .47 \\
\hline 85 & $7 / 26 / 00$ & $14: 20: 44$ & 116166.72 & .86 \\
\hline 80 & $7 / 26 / 00$ & $14: 20: 45$ & 14 & 7.36 \\
\hline 187 & $7 / 26 / 00$ & $14: 20: 47$ & 3.18 & .27 \\
\hline 0188 & $7 / 26 / 00$ & $14: 20: 48$ & 05 & .21 \\
\hline 0189 & $7 / 26 / 00$ & $14: 20: 50$ & 67.145 & .47 \\
\hline 0190 & $7 / 26 / 00$ & $14: 20: 51$ & 132946.2 & .29 \\
\hline 0191 & $7 / 26 / 00$ & $14: 20: 53$ & 120407.185 & .86 \\
\hline 0192 & $7 / 26 / 00$ & $14: 20: 54$ & 79.805 & .04 \\
\hline 0193 & $7 / 2$ & $14: 20: 56$ & .245 & \\
\hline 0194 & $7 / 26 / 00$ & $14: 20: 57$ & 119499. & .55 \\
\hline 20195 & $7 / 26 / 00$ & $14: 20: 59$ & 127626.835 & 3253.78 \\
\hline 0196 & $7 / 26 / 00$ & $14: 21: 00$ & 151609.215 & 3952.16 \\
\hline C0197 & $7 / 26 / 00$ & $14: 21: 02$ & 129411.52 & 3470.53 \\
\hline C0198 & $7 / 2$ & $14: 21: 03$ & 19.75 & .53 \\
\hline C0199 & $7 / 26 / 00$ & $14: 21: 05$ & 11.295 & .29 \\
\hline $\mathrm{C} 0200$ & $7 / 26 / 00$ & $14: 21: 06$ & 139663.26 & .87 \\
\hline C0201 & $7 / 26 / 00$ & $14: 21: 08$ & 09.92 & 3356.5 \\
\hline $\mathrm{C} 0202$ & $7 / 26 / 00$ & $14: 21: 09$ & 158833.99 & 4245.77 \\
\hline 3 & $7 / 26 / 00$ & $14: 21: 11$ & 148245.53 & .41 \\
\hline $\mathrm{CO} 204$ & $7 / 26 / 00$ & $14: 21: 12$ & 4.99 & \\
\hline C0205 & $7 / 26 / 00$ & $14: 21: 14$ & 155298.035 & 3606.37 \\
\hline C0206 & $7 / 26 / 00$ & $14: 21: 15$ & 131974.565 & 4077.87 \\
\hline $\mathrm{C} 0207$ & $7 / 26 / 00$ & $14: 21: 17$ & 152874.005 & 3545.75 \\
\hline $\mathrm{C} 0208$ & $7 / 26 / 00$ & $14: 21: 18$ & 140162.25 & 3674.16 \\
\hline 0209 & $7 / 26 / 00$ & $14: 21: 20$ & 67.435 & 3390.7 \\
\hline C0210 & $7 / 26 / 00$ & $14: 21: 21$ & 163710.015 & 4145. \\
\hline $\mathrm{C} 0211$ & $7 / 26 / 00$ & $14: 21: 23$ & 168690.08 & 3924.32 \\
\hline 212 & $7 / 26 / 00$ & $14: 21: 24$ & 151770.43 & 4137.44 \\
\hline & $7 / 26 / 00$ & $14: 21: 26$ & 163250.345 & 4329.52 \\
\hline & J & $21: 27$ & 152188.715 & 3957. \\
\hline & $7 / 26 / 00$ & $14: 21: 29$ & 158238.09 & 3812.46 \\
\hline & & $14: 21: 30$ & 146314.965 & 3346.6 \\
\hline
\end{tabular}




\begin{tabular}{|c|c|c|c|c|}
\hline 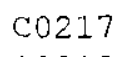 & $\pi_{2}$ & 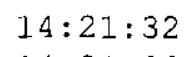 & & \\
\hline & $7 / 26 / 00$ & $14: 21: 33$ & 168503.105 & 4529.34 \\
\hline 19 & $6 / 00$ & $14: 21: 35$ & 151798.68 & \\
\hline 220 & $7 / 26 / 00$ & $14: 21: 36$ & 157454.645 & \\
\hline 221 & $7 / 26 / 00$ & $14: 21: 38$ & 168957.415 & 3625 \\
\hline 0222 & $7 / 26 / 00$ & $14: 21: 39$ & 172874.08 & 2 \\
\hline 0223 & $7 / 26 / 00$ & $14: 21: 41$ & 167649.375 & \\
\hline 224 & $7 / 26 / 00$ & $14: 21: 42$ & 162893.385 & \\
\hline 225 & $7 / 26 / 00$ & $14: 21: 44$ & 178150.055 & \\
\hline 226 & $7 / 26 / 00$ & $14: 21: 45$ & 166065.245 & 31280 \\
\hline 227 & $7 / 26 / 00$ & $14: 21: 47$ & 142444.3 & 3563 \\
\hline Co228 & $7 / 26 / 00$ & $14: 21: 48$ & 171 & \\
\hline 229 & $7 / 26 / 00$ & $14: 21: 50$ & 184295.265 & \\
\hline 230 & $7 / 26 / 00$ & $14: 21: 51$ & 155736.24 & \\
\hline 0231 & $7 / 26 / 00$ & $14: 21: 53$ & 172319.215 & \\
\hline $\mathrm{C} 0232$ & $7 / 26 / 00$ & $14: 21: 54$ & 147415.29 & \\
\hline 0233 & $7 / 26 / 00$ & $14: 21: 56$ & 167203.59 & .82 \\
\hline 0234 & $7 / 26 / 00$ & $14: 21: 57$ & 159720.625 & \\
\hline 235 & $7 / 26 / 00$ & $14: 21: 59$ & 178042.84 & 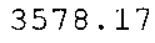 \\
\hline 0236 & $7 / 26 / 00$ & $14: 22: 00$ & 171598.065 & \\
\hline $\mathrm{C} 0237$ & $7 / 26 / 00$ & $14: 22: 02$ & 7.655 & .45 \\
\hline $\mathrm{C} 0238$ & $7 / 26 / 00$ & $14: 22: 03$ & 6.195 & \\
\hline $\mathbf{C} 0239$ & $7 / 26 / 00$ & $14: 22: 05$ & 4.305 & 03 \\
\hline 0240 & $7 / 26 / 00$ & $14: 22: 06$ & 164492.695 & .8 \\
\hline $\mathrm{C} 0241$ & $7 / 26 / 00$ & $14: 22: 08$ & 7.525 & 37 \\
\hline $\mathrm{CO} 242$ & $7 / 26 / 00$ & $14: 22: 09$ & 32.265 & \\
\hline $\mathrm{C} 0243$ & $7 / 26 / 00$ & $14: 22: 11$ & .335 & \\
\hline 0244 & $7 / 26 / 00$ & $14: 22: 12$ & 7.585 & .07 \\
\hline 0245 & $7 / 26 / 00$ & $14: 22: 14$ & 159163.525 & .15 \\
\hline$C 0246$ & $7 / 26 / 00$ & $14: 22: 15$ & 1.695 & 3. \\
\hline $\mathrm{C} 0247$ & $7 / 26 / 00$ & $14: 22: 17$ & 7.175 & 01 \\
\hline C0248 & $7 / 2$ & $14: 22: 18$ & 17 & \\
\hline $\mathrm{C} 0249$ & $7 / 26 / 00$ & $14: 22: 20$ & 177 & 13 \\
\hline $\mathrm{C} 0250$ & $7 / 26 / 00$ & $14: 22: 21$ & 184429.085 & 9.44 \\
\hline C0251 & $7 / 26 / 00$ & $14: 22: 23$ & 179522.58 & 5.8 \\
\hline $\mathrm{C} 0252$ & $7 / 26 / 00$ & $14: 22: 24$ & 184 & .56 \\
\hline $\mathrm{C} 0253$ & $7 / 26 / 00$ & $22: 26$ & 3.03 & 94 \\
\hline C0254 & $7 / 26 / 00$ & $14: 22: 27$ & 5.815 & 96 \\
\hline $\mathrm{C} 0255$ & $7 / 26 / 00$ & $14: 22: 29$ & 161926.245 & .07 \\
\hline C0256 & $7 / 26 / 00$ & $14: 22: 30$ & 168892.28 & .25 \\
\hline $\mathrm{C} 0257$ & $7 / 26 / 00$ & $14: 22: 32$ & 5.18 & 4248.21 \\
\hline $\mathrm{C} 0258$ & $7 / 26 / 00$ & $14: 22: 33$ & .765 & 29 \\
\hline $\mathrm{C} 0259$ & $7 / 26 / 00$ & $14: 22: 35$ & 2.885 & 91 \\
\hline $\mathrm{C} 0260$ & $7 / 26 / 00$ & $14: 22: 36$ & 156123.48 & 3984.12 \\
\hline $\mathrm{C} 0261$ & $7 / 26 / 00$ & $14: 22: 38$ & 164421.875 & 3156.62 \\
\hline $\mathrm{C} 0262$ & $7 / 26 / 00$ & $14: 22: 39$ & 164761.08 & .48 \\
\hline $\mathrm{C} 0263$ & $7 / 26 / 00$ & $14: 22: 41$ & 8.185 & 7.75 \\
\hline $\mathrm{C} 0264$ & $7 / 26 / 00$ & $14: 22: 42$ & 163573.295 & 3747.65 \\
\hline $\mathrm{C} 0265$ & $7 / 26 / 00$ & $14: 22: 44$ & 169261.885 & 734 \\
\hline 266 & $7 / 26 / 00$ & $14: 22: 45$ & 156479.39 & 4183.04 \\
\hline $\mathrm{C} 0267$ & $7 / 26 / 00$ & $14: 22: 47$ & 154507.25 & .77 \\
\hline $\mathrm{C} 0268$ & $7 / 26 / 00$ & $14: 22: 48$ & 151422.61 & 2974.95 \\
\hline 269 & $7 / 26 / 00$ & $14: 22: 50$ & 158573.79 & 4609.81 \\
\hline 270 & $7 / 26 / 00$ & $14: 22: 51$ & 150028.86 & \\
\hline $\mathrm{C} 0271$ & $7 / 26 / 00$ & $14: 22: 53$ & 159201.615 & 3683.31 \\
\hline & $7 / 26 / 00$ & $: 22: 54$ & 159840.71 & 3924. \\
\hline
\end{tabular}




\begin{tabular}{|c|c|c|c|c|}
\hline & & & & \\
\hline & $7 / 26 / 00$ & & & \\
\hline & & 59 & .57 & \\
\hline & & $23: 00$ & 15 & 8. \\
\hline 277 & $26 / 00$ & $14: 23: 02$ & 127910.005 & 874.96 \\
\hline 278 & $7 / 26 / 00$ & $14: 23: 03$ & 137019.17 & 50706 \\
\hline 79 & $26 / 00$ & $: 23: 05$ & 25 & \\
\hline & & $: 23: 06$ & 305 & \\
\hline & $6 / 00$ & $4: 23: 08$ & 18.475 & 1.63 \\
\hline $2 \varepsilon$ & $7 / 26 / 00$ & $14: 23: 09$ & 166939.615 & \\
\hline 128 & $26 / 00$ & $23: 11$ & 75 & \\
\hline & & $23: 12$ & 685 & \\
\hline & 10 & $:: 23: 14$ & 22.79 & \\
\hline 86 & $26 / 00$ & $4: 23: 15$ & 85.765 & \\
\hline $7+2$ & $7 / 26 / 00$ & $14: 23: 17$ & & .65 \\
\hline 288 & $/ 26 / 00$ & $14: 23: 18$ & 14 & 24 \\
\hline 89 & $26 / 00$ & $4: 23: 20$ & 5 & \\
\hline & $7 / 26 / 00$ & $3: 21$ & .91 & \\
\hline & $7 / 26 / 00$ & $14: 23: 23$ & 725 & $24+2$ \\
\hline 2 & $7 / 26 / 00$ & $14: 23: 24$ & & \\
\hline & $/ 26 / 00$ & $14: 23: 26$ & & \\
\hline 02 & 10 & $3: 27$ & 65 & .11 \\
\hline 95 & $26 / 00$ & $4: 23: 29$ & 54.505 & $\cdots 1$ \\
\hline & & 0 & & \\
\hline & $7 / 26 / 00$ & $14: 23: 32$ & & \\
\hline 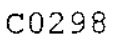 & $7 / 2$ & $3: 33$ & 45 & \\
\hline & $7 / 2$ & $14: 23: 35$ & 35 & ? \\
\hline 00 & $7 / 26 /$ & $4: 23: 36$ & .75 & 1.15 \\
\hline 1 & $7 / 2$ & $3: 38$ & 95 & \\
\hline & 0 & & & \\
\hline 0 & $7 / 26$ & 11 & & \\
\hline 0 & $7 / 26 / 00$ & $3: 42$ & 65 & 3.01 \\
\hline 05 & $7 / 26 / 00$ & $4: 23: 44$ & & 1.7 \\
\hline $\mathrm{CO} 3$ & $7 / 26 / 00$ & $14: 23: 45$ & 585 & .34 \\
\hline & 0 & & & 16 \\
\hline $0:$ & $7 / 26$ & $3: 48$ & & 81 \\
\hline & $7 / 26 / 00$ & $4: 23: 50$ & 95 & .18 \\
\hline 03 & $7 / 26 / 00$ & $14: 23: 51$ & 65 & 59 \\
\hline C03 & $7 / 26 / 00$ & 14: & & .81 \\
\hline & $7 /$ & 4 & & \\
\hline & $7 / 2$ & $: 56$ & 45 & 35 \\
\hline & $7 / 26 / 00$ & $3: 57$ & 1.33 & 2.86 \\
\hline$C$ & $7 / 26 / 00$ & $14: 23: 59$ & 7.11 & 2.66 \\
\hline & $7 / 26 / 00$ & $14: 24: 00$ & 391.865 & 3798.1 \\
\hline $\mathrm{Cl}$ & $7 / 26 / 00$ & & 10.545 & 1072.82 \\
\hline & & & & \\
\hline & $7 / 26 / 00$ & 5 & 10 & 2.58 \\
\hline & $7 / 26 / 00$ & 4. & 7.475 & .99 \\
\hline & $7 / 26 / 00$ & $14: 24: 08$ & 7.315 & 3736.6 \\
\hline $\mathrm{CO}$ & $7 / 26 / 00$ & $14: 24: 09$ & 1. & 3774.43 \\
\hline & $7 / 26 / 00$ & & & \\
\hline & $7 / 26 / 00$ & & 12 & 6.31 \\
\hline & $7 / 26 / 00$ & $14: 24: 14$ & 120579.055 & 3803.97 \\
\hline & $7 / 26 / 00$ & $14: 24: 15$ & $12 ?$ & \\
\hline & $7 / 26 / 00$ & $4: 17$ & & \\
\hline & 712610 & & (2) 8 & 3598. \\
\hline
\end{tabular}




\begin{tabular}{|c|c|c|c|c|}
\hline 9 & & 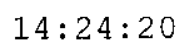 & 122835.45 & 2929.5 \\
\hline 30 & $7 / 26 / 00$ & $14: 24: 21$ & 128744.795 & 2916.39 \\
\hline & 00 & $14: 24: 23$ & 116554.905 & 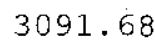 \\
\hline & & $: 24: 24$ & 013.915 & 698.84 \\
\hline & 100 & $14: 24: 26$ & 4399.96 & .63 \\
\hline 334 & $7 / 26 / 00$ & $14: 24: 27$ & 105813.92 & 946.51 \\
\hline 5 & $/ 26 / 00$ & $14: 24: 29$ & 1202 & 170 \\
\hline & $26 / 00$ & $14: 24: 30$ & 121567.47 & 0526 \\
\hline 337 & $26 / 00$ & $1: 24: 32$ & 8099.885 & 54.7 \\
\hline 338 & $/ 26 / 00$ & $4: 24: 33$ & 134830.765 & 049.35 \\
\hline 0339 & $7 / 26 / 00$ & $14: 24: 35$ & 129662.3 & \\
\hline 0340 & $7 / 26 / 00$ & $: 36$ & 126 & \\
\hline 41 & $26 / 00$ & $1: 24: 38$ & 26 & . \\
\hline 2 & $/ 26 / 00$ & $4: 24: 39$ & 1022 & \\
\hline 343 & $7 / 26 / 00$ & $4: 24: 41$ & 1094 & 64.29 \\
\hline 0344 & $7 / 26 / 00$ & $14: 24: 42$ & & \\
\hline 345 & $7 / 26 / 00$ & $14: 24: 44$ & 55 & .58 \\
\hline 6 & $7 / 26 / 00$ & $4: 24: 45$ & 45 & \\
\hline & $7 / 26 /$ & 47 & 35 & \\
\hline 8 & $7 / 26 / 00$ & 48 & .795 & \\
\hline 349 & $7 / 26 / 00$ & & & \\
\hline 03 & $7 / 26$ & 51 & 05 & \\
\hline 1 & $7 / 26 /$ & 53 & 35 & .29 \\
\hline & 1720 & 54 & & \\
\hline C0353 & $7 / 26 / 00$ & $14: 24: 56$ & 132 & .41 \\
\hline & $7 / 26 / 00$ & & & \\
\hline 5 & $7 / 26 / 00$ & 58 & & 5.09 \\
\hline 6 & $7 / 26 / 00$ & 00 & 102 & 9.46 \\
\hline 7 & $7 / 26 / 00$ & $14: 25: 02$ & 31 & \\
\hline 0358 & $7 / 26 / 00$ & $14: 25: 03$ & 118 & \\
\hline & $7 / 2$ & & 55 & 49 \\
\hline U & $7 / 26 / 00$ & 06 & 5 & 9.98 \\
\hline 61 & $7 / 26 / 00$ & 07 & 21 & 9.99 \\
\hline C & $7 / 26 / 00$ & $14:$ & 11 & 1.78 \\
\hline C0363 & $7 / 26 / 00$ & $14: 25: 11$ & 123 & 1.28 \\
\hline & $7 / 26$ & 12 & & \\
\hline & $7 / 26$ & 13 & 15 & 44 \\
\hline & $7 / 26 / 00$ & 15 & 32 & 1.93 \\
\hline $\mathrm{C} 0367$ & $7 / 26 / c$ & $14: 25: 16$ & 4 & 9.69 \\
\hline $\mathrm{CO}$ & $7 / 26 / 00$ & $14: 25: 18$ & .385 & 2882.38 \\
\hline & & & & 179.37 \\
\hline & $7 / 2$ & 21 & & 1.8 \\
\hline & $7 / 26 / 00$ & $: 25$ & 6 & 5.45 \\
\hline $\mathrm{C} 0372$ & $7 / 26 / 00$ & $14: 25: 24$ & 113589.155 & 2876.32 \\
\hline C0373 & $7 / 26 / 00$ & 14: & 4.25 & 649.8 \\
\hline & & & & 7021 \\
\hline & $7 / 26$ & & & 9 \\
\hline & $7 / 26 / 00$ & $4: 25: 30$ & 59 & 5.79 \\
\hline C0377 & $7 / 26 / 00$ & $4: 25: 31$ & 1049 & 3570.82 \\
\hline C0378 & $7 / 26 / 00$ & $14: 25: 33$ & $10 s$ & 3716.36 \\
\hline & $7 / 26 / 00$ & & & 8472 \\
\hline & $7 / 26 / 00$ & 1.25 .36 & 225 & 731 \\
\hline & $7 / 26 / 00$ & $14: 25: 37$ & 89329.145 & 3434.5 \\
\hline $\mathrm{CO}$ & $7 / 26 / 00$ & $: 25: 39$ & 4.71 & 3078.79 \\
\hline & 00 & $: 25: 41$ & 1.145 & \\
\hline & $1 / 26 / 00$ & & 105785.28 & 3708.63 \\
\hline
\end{tabular}




$\begin{array}{lllll}\mathrm{C} 0385 & 7 / 26 / 00 & 14: 25: 43 & 105949.91 & 3623.08 \\ \mathrm{C} 0386 & 7 / 26 / 00 & 14: 25: 45 & 101188.605 & 3808.42 \\ \mathrm{C} 0387 & 7 / 26 / 00 & 14: 25: 46 & 110270.715 & 3668.96 \\ \mathrm{C} 0388 & 7 / 26 / 00 & 14: 25: 48 & 106238.025 & 3523.73 \\ \mathrm{C} 0389 & 7 / 26 / 00 & 14: 25: 49 & 120052.21 & 4215.8 \\ \mathrm{C} 0390 & 7 / 26 / 00 & 14: 25: 51 & 113486.305 & 3250.56 \\ \mathrm{C} 0391 & 7 / 26 / 00 & 14: 25: 52 & 118484.13 & 2852.43 \\ \mathrm{C} 0392 & 7 / 26 / 00 & 14: 25: 54 & 115277.935 & 3949.45 \\ \mathrm{C} 0393 & 7 / 26 / 00 & 14: 25: 55 & 100861.075 & 3660.05 \\ \mathrm{C} 0394 & 7 / 26 / 00 & 14: 25: 57 & 116347.775 & 3464.92 \\ \mathrm{C} 0395 & 7 / 26 / 00 & 14: 25: 58 & 111781.19 & 3332.22 \\ \mathrm{C} 0396 & 7 / 26 / 00 & 14: 26: 00 & 127277.235 & 2979.81 \\ \mathrm{C} 0397 & 7 / 26 / 00 & 14: 26: 01 & 108375.85 & 3124.18 \\ \mathrm{C} 0398 & 7 / 26 / 00 & 14: 26: 03 & 112246.315 & 3239.85 \\ \mathrm{C} 0399 & 7 / 26 / 00 & 14: 26: 04 & 101143.04 & 3260.8 \\ \mathrm{C} 0400 & 7 / 26 / 00 & 14: 26: 06 & 104535.7 & 3409.71\end{array}$

\section{Revision 0}




\section{APPENDIX C - TEST INCIDENT REPORT}

Incident Identification

Incident No.

Date

Test Case No.

Item No.

Description of Incident: (Identify expected results and actual results)

\section{Determination}

[ ]Fix Before Implementation [ ]Fix After Implementation [ ]Change in Scope Action Taken:

Impact On Previous or Following Tests:

Incident Documented:

Test Performer

Date

Test Witness

Date

Test Administrator

Date

\section{Correction Verified:}

Test Performer

Date

Test Witness

Date 


\section{APPENDIX D - TEST INCIDENT REPORTS}

Incident reports, if any will be generated at the conclusion of testing. 


\subsection{SUMMARY}

Evaluate and summarize the test results. Identify the system and the items tested, indicating their revision identification. Indicate the environment in which the testing activities took place. State whether or not the system is ready for operation. Supply references to the following documentation if they exist: test plan, test design, test procedures, test item transmittal reports, test logs, and test incident report.

\subsection{VARIANCES}

Report any variances of the test items from their design specifications. Indicate any variances from the test plan, test designs, or test procedures during the testing. Specify the reason for each variance.

\subsection{COMPREHENSIVE ASSESSMENT}

Evaluate the comprehensiveness of the testing process against the acceptance criteria. Identify features or feature combinations that were not sufficiently tested, and explain the reasons.

\subsection{SUMMARY OF RESULTS}

Summarize the results of testing. Identify all resolved incidents, and summarize their resolutions. Identify all unresolved incidents and the impact on placing the system into operation.

\subsection{EVALUATION}

Provide an overall evaluation of each test item, based upon the test results. Provide an estimate of failure risk.

\subsection{SUMMARY OF ACTIVITIES}

Summarize the major testing activities and events. Summarize resource consumption data, for example, total staffing level, total machine time, and total elapsed time used for each of the major testing activities. 
HNF-6995

$09 / 13 / 2000$

7.0 APPROVALS

Revision 0

Specify the names and titles of all persons who must approve this report. Provide space for signatures and dates.

Date

Date

Date 